\title{
Infrared Catastrophe for Nelson's Model -Non-Existence of Ground State and Soft-Boson Divergence-
}

\author{
By
}

Masao HiroKawA*

\begin{abstract}
We mathematically study the infrared catastrophe for the Hamiltonian of Nelson's model when it has the external potential in a general class. For the model, we prove the pull-through formula on ground states in operator theory first. Based on this formula, we show both non-existence of any ground state and divergence of the total number of soft bosons.
\end{abstract}

\section{$\S 1 . \quad$ Introduction}

The purpose of the present paper is to investigate mathematically the infrared (IR) catastrophe for Nelson's Hamiltonian [25], in particular nonexistence of ground state and the divergence of the total number of soft bosons (soft-boson divergence). The exact definition of ground state will be stated in $\S 2$. The definition of soft boson will be explained later. IR catastrophe is the trouble of IR divergence caused by massless particles forming a quantized field. Nelson's Hamiltonian is the Hamiltonian of the so-called Nelson's model describing a system of a quantum particle, which moves in the 3-dimensional Euclidean space $\mathbb{R}^{3}$ under the influence of an external potential, and which interacts with a massless scalar Bose field. The massless scalar Bose field is the quantized scalar field made of massless bosons. The boson is the (quantum)

Communicated by T. Kawai. Received April 7, 2004. Revised October 14, 2004, June 1, 2005, September 5, 2005.

2000 Mathematics Subject Classification(s): 81T10, 81V10.

This work is supported by JSPS, Grant-in-Aid for Scientific Research (C) 16540155.

* Department of Mathematics, Okayama University, 700-8530 Okayama, Japan.

e-mail: hirokawa@math.okayama-u.ac.jp 
particle following the Bose-Einstein statistics. In the present paper the soft boson means the boson in a ground state.

Recently, the spectral properties of Nelson's Hamiltonian has been studied rather intensively (e.g., [2, 9, 11, 16, 20, 24]). In particular, Betz et al. showed in [9] that when the external potential is in the Kato class the total number of soft bosons for Nelson's Hamiltonian diverges under the infrared singularity (IRS) condition. We will concretely define this condition in $\S 2$. Around the same time Lörinczi et al. showed in [24] that when the external potential is strongly confining there is no ground state of Nelson's Hamiltonian in spatial dimension 3. The results in both [9] and [24] are proved by means of functional integrals.

In [11] Deresiński and Gérard treated the problem of non-existence of ground state by $L^{2}$-theoretical method and proved the non-existence of any ground state for Nelson's Hamiltonian under the assumption that the external potential is strongly confining. They employed an amazingly simple method based on the $L^{2}$-theoretical pull-through formula. However, the results shown in [11] do not seem to include the case of decaying potentials such as the Coulomb potential. For another model, the so-called Pauli-Fierz model [26], it was clarified in $[8,14]$ that there exists a ground state even under IRS condition, when Pauli-Fierz's Hamiltonian has the Coulomb-type potential.

In the present paper we consider Nelson's Hamiltonian with a general class of potentials including both strongly confining potentials and Coulombtype potentials and prove in a unified way the non-existence of any ground state and the soft-boson divergence. Following the methods in [11, 24] to prove the non-existence of any ground state, we are required to invent some suitable technique in order to include Coulomb-type potentials. Thus, the present paper looks at the problem from a different angle. Following the physical observation stated below, we adopt an operator-theoretical method in which we combine the technique of spatial localization presented by Griesemer, Lieb, and Loss [14] and an approach based on the proof of the absence of ground state by Arai, Hiroshima, and the author [6]. We believe that this approach is new.

In this paper the operator-theoretical pull-through formula announced in [17] plays a crucial role. So, we give a complete version of its proof. To the best of author's knowledge, the approach presented in this paper is the first to establish the pull-through formula in an operator-theoretical framework. Such an operator-theoretical formula makes it possible to analyze infrared catastrophe in mathematical detail $[7,19,21]$. In physics it is generally expected that the non-existence of ground state results from the soft-boson divergence. 
From a mathematical point of view, however, we establish in the present paper that the pull-through formula implies both the non-existence of ground state (Theorems 2.1 and 2.2) and the soft-boson divergence (Theorems 2.3 and 2.4), independently to each other.

In a mathematical treatment, this IR problem was first studied for a fermion-boson model related to Nelson's by Fröhlich [12]. It is worthy of note that Pizzo developed Fröhlich's work in [27]. We tackled IR problem of proving the non-existence of ground state for the so-called generalized spin-boson (GSB) model from an operator-theoretical point of view in [6], while we studied a mathematical mechanism of existence of ground states for it in [4]. However, because GSB model is very general, the information on IR problem for it was so limited that we could not entirely achieve our goal. In the present paper, we completely achieve it for Nelson's Hamiltonian with the external potential in the general class.

For our goal, we present the following physical image of the relation between the soft-boson divergence and the non-existence of ground state: To begin with, the quantum particle coupled with the field formed by bosons is generally dressed in the cloud of bosons, which makes the so-called quasi-particle. In particular, the total number of soft bosons for Nelson's model diverges under IRS condition. So, if a ground state exists under IRS condition, then the quantum particle has to dress itself in the cloud of infinitely many soft bosons. Thus, we can hardly expect that the cloud is spatially localized into a finite area. Namely, because the soft boson is the boson in a ground state, the uncertainty of the particle's position in the ground state must be infinite under IRS condition. On the other hand, once a ground state exists, we can generally expect to obtain the finite uncertainty of the position in the ground state in order to observe the particle's position. Therefore, the existence of a ground state of Nelson's model under IRS condition must imply a contradiction in quantum theory. We seek to express this image in a mathematical way.

The present paper is organized as follows. In $\S 2$ we state main results. On the external potential we impose two kind of assumptions, assumption (A) and assumption (C). The assumption (A) is of rather general nature. Assuming (A), we assert that ground states are absent from the domain of the square of position operator (Theorem 2.1). Assumption (C) is more concrete and more restrictive than (A). Assuming (C), we establish the non-existence of any ground state (Theorem 2.3). Theorems 2.2 and 2.4 are concerned with estimates of number of soft bosons. In $\S 3$ the operator-theoretical pull-through formula is proved and a useful identity is derived from it. In $\S 4$ we prove Theorem 2.1 
and in $\S 5$ Theorem 2.3. In $\S 6$ the finite uncertainty is argued, and combining this with the absence theorem and the estimate proved in $\S 4$, we establish our final results, Theorems 2.2 and 2.4 .

\section{$\S 2 . \quad$ Main Results}

The position of the quantum particle with mass $m=1$ is denoted by $x$, the momentum by $p:=-i \nabla_{x}$. Here we employ the natural units. Namely, we set $\hbar=1, c=1$ throughout. As the Hamiltonian for the quantum particle, we consider the Schrödinger operator acting in $L^{2}\left(\mathbb{R}^{3}\right)$,

$$
H_{\mathrm{at}}:=\frac{1}{2} p^{2}+V
$$

with an external potential $V$.

We consider two types of assumption for $H_{\text {at }}$ as the notice was given in $\S 1$, i.e., general assumption (A) and concrete assumption (C). We prove under (A) that any ground state is not in the subspace characterized by a kind of spatial localization (Theorem 2.1). Under (C) we completely prove the non-existence of any ground state (Theorem 2.2).

(A) $H_{\text {at }}$ is a self-adjoint operator bounded from below such that $D\left(H_{\text {at }}\right) \subset$ $D\left(p^{2}\right)$. Moreover, $H_{\text {at }}$ has a ground state $\psi_{\text {at }}$.

Here $D(T)$ denotes the domain of an operator $T$. We denote the ground state energy by $E_{\text {at }}:=\inf \sigma\left(H_{\text {at }}\right)$, where $\sigma(T)$ denotes the spectrum of a closed operator $T$.

For completion of the non-existence theorem, we investigate the following two classes of external potentials. The two classes include the strongly confining potential, long and short range ones.

(C1) [2]:

(C1-1) $H_{\text {at }}$ is self-adjoint on $D\left(H_{\text {at }}\right)=D\left(p^{2}\right) \cap D(V)$ and bounded from below,

(C1-2) there exist positive constants $c_{1}$ and $c_{2}$ such that $|x|^{2} \leq c_{1} V(x)+c_{2}$ for almost every (a.e.) $x \in \mathbb{R}^{3}$, and $\int_{|x| \leq R}|V(x)|^{2} d^{3} x<\infty$ for all $R>0$.

(C2) [31]:

(C2-1) $V \in L^{2}\left(\mathbb{R}^{3}\right)+L^{\infty}\left(\mathbb{R}^{3}\right)$, and $\lim _{|x| \rightarrow \infty}|V(x)|=0$. 
In this case, by Kato's theorem [29, Theorem X15] and the well-known fact [30, $\S$ XIII.4, Example 6], we have the following:

Proposition 2.1. Assume (C2-1). Then,

(i) $H_{\text {at }}$ is self-adjoint on $D\left(p^{2}\right)$.

(ii) $V$ is infinitesimally $p^{2}$-bounded.

(iii) $\sigma_{\mathrm{ess}}\left(H_{\mathrm{at}}\right)=[0, \infty)$, where $\sigma_{\mathrm{ess}}\left(H_{\mathrm{at}}\right)$ is the essential spectrum of $H_{\mathrm{at}}$.

We assume the following in addition to (C2-1):

(C2-2) $H_{\text {at }}$ has a ground state $\psi_{\text {at }}$ satisfying $\psi_{\text {at }}(x)>0$ for a.e. $x \in \mathbb{R}^{3}$ and $E_{\text {at }}<0$.

Both in $(\mathrm{C} 1)$ and $(\mathrm{C} 2)$, condition $(\mathrm{A})$ holds and we have a ground state $\psi_{\text {at }}$ of $H_{\text {at. }}$. We say that $V$ is in (C1) (resp. (C2)) if (C1-1) and (C1-2) (resp. (C2-1) and (C2-2)) hold.

Our quantum particle is coupled with a massless scalar Bose field. We first prepare some notations for the quantized field. For the state space of scalar bosons, we take the Hilbert space given by the symmetric Fock space $\mathcal{F}:=$ $\bigoplus_{n=0}^{\infty}\left[\otimes_{\mathrm{s}}^{n} L^{2}\left(\mathbb{R}^{3}\right)\right]$ over $L^{2}\left(\mathbb{R}^{3}\right)$, where $\otimes_{\mathrm{s}}^{n} L^{2}\left(\mathbb{R}^{3}\right)$ denotes the $n$-fold symmetric tensor product of $L^{2}\left(\mathbb{R}^{3}\right)$, the space of all square-integrable functions, and $\otimes_{\mathrm{s}}^{0} L^{2}\left(\mathbb{R}^{3}\right):=\mathbb{C}$. The finite particle space $\mathcal{F}_{0}$ is defined by $\mathcal{F}_{0}:=\{\Psi=$ $\Psi^{(0)} \oplus \cdots \oplus \Psi^{(n)} \oplus \cdots \in \mathcal{F} \mid \Psi^{(n)}=0$ for $\left.n \geq \exists n_{0}\right\}$. For every $f \in L^{2}\left(\mathbb{R}^{3}\right)$ and $\Psi=\Psi^{(0)} \oplus \Psi^{(1)} \oplus \cdots \oplus \Psi^{(n)} \oplus \cdots \in \mathcal{F}_{0}$, the smeared annihilation operator $a(f)$ of bosons is defined by

$$
(a(f) \Psi)^{(n)}\left(k_{1}, \cdots, k_{n}\right):=\sqrt{n+1} \int_{\mathbb{R}^{3}} f(k)^{*} \Psi^{(n+1)}\left(k, k_{1}, \cdots, k_{n}\right) d^{3} k
$$

as $\otimes_{\mathrm{s}}^{n+1} L^{2}\left(\mathbb{R}^{3}\right) \ni \Psi^{(n+1)} \rightarrow(a(f) \Psi)^{(n)} \in \otimes_{\mathrm{s}}^{n} L^{2}\left(\mathbb{R}^{3}\right)$ for $n=0,1,2, \cdots$, where $f(k)^{*}$ is the complex conjugate of $f \in L^{2}\left(\mathbb{R}^{3}\right)$. Then, $a(f)$ is closable for every $f \in L^{2}\left(\mathbb{R}^{3}\right)$. We denote its closure by the same symbol. We define the smeared creation operator $a^{\dagger}(f)$ by the adjoint operator of $a(f)$, i.e., $a^{\dagger}(f)=a(f)^{*}$, for every $f \in L^{2}\left(\mathbb{R}^{3}\right)$.

The smeared annihilation and creation operators satisfy the standard canonical commutation relations $(\mathrm{CCR})$ :

$$
\begin{gathered}
{\left[a(f), a^{\dagger}(g)\right]=(f, g)_{L^{2}} \equiv \int_{\mathbb{R}^{3}} f(k)^{*} g(k) d^{3} k,} \\
{[a(f), a(g)]=0, \quad\left[a^{\dagger}(f), a^{\dagger}(g)\right]=0, \quad \forall f, g \in L^{2}\left(\mathbb{R}^{3}\right),}
\end{gathered}
$$


on $\mathcal{F}_{0}$.

In this paper, we consider the following dispersion relation $\omega(k)$,

$$
\omega(k)=|k| .
$$

Then the free field energy operator $H_{\mathrm{f}}$ is the second quantization of $\omega$, i.e.,

$$
H_{\mathrm{f}}:=d \Gamma(\omega)
$$

Here, for a self-adjoint operator $h$ acting in $L^{2}\left(\mathbb{R}^{3}\right)$, its second quantization is defined by

$$
d \Gamma(h):=\bigoplus_{n=0}^{\infty} h^{(n)}
$$

where $h^{(n)}$ is the closure of $\sum_{j=1}^{n} I \otimes \cdots \otimes \stackrel{h}{h}_{j \text { th }} \otimes \otimes I \equiv h \otimes I \otimes \cdots \otimes I+I \otimes$ $h \otimes I \otimes \cdots \otimes I+\cdots+I \otimes \cdots \otimes I \otimes h$, i.e.,

$$
h^{(n)}:=\overline{\sum_{j=1}^{n} I \otimes \cdots \otimes \underset{j \text {-th }}{h} \otimes \cdots \otimes I}
$$

acting in $\otimes_{\mathrm{s}}^{n} L^{2}\left(\mathbb{R}^{3}\right)$, where $I$ denotes the identity operator on $L^{2}\left(\mathbb{R}^{3}\right)$, and $h^{(0)}=0$. We note that $d \Gamma(h)$ is a self-adjoint operator acting in $\mathcal{F}$. Thus, for $H_{\mathrm{f}}$ we employed the multiplication operator $\omega$ as $h$ in (2.2). We define the subspace $\mathcal{F}(\omega)$ by the linear hull of $\left\{\Omega_{0}, a^{\dagger}\left(f_{1}\right) \cdots a^{\dagger}\left(f_{\nu}\right) \Omega_{0} \mid \nu \in \mathbb{N}, f_{j} \in D(\omega)\right.$, $j=1, \cdots, \nu\}$, where $\Omega_{0}$ is the Fock vacuum, i.e.,

$$
\Omega_{0}=1 \oplus 0 \oplus 0 \oplus \cdots \in \mathcal{F} .
$$

Then, the action of $H_{\mathrm{f}}$ is given by

$$
\otimes_{\mathrm{s}}^{n} L^{2}\left(\mathbb{R}^{3}\right) \ni\left(H_{\mathrm{f}} \Psi\right)^{n}\left(k_{1}, \cdots, k_{n}\right)=\sum_{j=1}^{n}\left|k_{j}\right| \Psi^{(n)}\left(k_{1}, \cdots, k_{n}\right), \quad \forall n \in \mathbb{N},
$$

and $\left(H_{\mathrm{f}} \Psi\right)^{(0)}=0$ for $\Psi=\Psi^{(0)} \oplus \Psi^{(1)} \oplus \cdots \in \mathcal{F}(\omega)$. $H_{\mathrm{f}}$ is symbolically written as

$$
H_{\mathrm{f}}=\int_{\mathbb{R}^{3}}|k| a^{\dagger}(k) a(k) d^{3} k
$$

using symbolical representation of the annihilation operator by the kernel $a(k)$,

$$
a(f)=\int_{\mathbb{R}^{3}} a(k) f(k)^{*} d^{3} k .
$$

We note that such symbolical notations are often used in physics. 
Remark 1. Fix $k \in \mathbb{R}^{3}$ arbitrarily. Then, the symbolic kernel $a(k)$ of the annihilation operator is given by

$$
(a(k) \Psi)^{(n)}\left(k_{1}, \cdots, k_{n}\right):=\sqrt{n+1} \Psi^{(n+1)}\left(k, k_{1}, \cdots, k_{n}\right)
$$

for $n=0,1,2, \cdots$. We note that $a(k)$ is well-defined as an operator for $\Psi \in$ $D_{\mathcal{S}}:=\left\{\Psi=\Psi^{(0)} \oplus \cdots \oplus \Psi^{(n)} \oplus \cdots \in \mathcal{F}_{0} \mid \Psi^{(n)} \in \mathcal{S}\left(\mathbb{R}^{3}\right), n \in \mathbb{N}\right\}$, where $\mathcal{S}\left(\mathbb{R}^{3}\right)$ is the set of all functions in the Schwartz class. The kernel $a(k)$ is defined pointwise by (2.3), so that a certain kind of continuity is required for $\Psi$. See, for example, $[1, \S 2.2]$ and $[3, \S 8-3]$. It is well known that $a(k)^{*}$ is not densely defined $\left[29, \S\right.$ X.7]; indeed, $a(k)^{*}$ is trivial [3, Proposition 8.2], i.e., $D\left(a(k)^{*}\right)=\{0\}$, so that $a(k)$ is not closable by [28, Theorem VIII.1(b)].

The Hilbert space in which the Hamiltonian of Nelson's model acts is defined by $\mathcal{H}:=L^{2}\left(\mathbb{R}^{3}\right) \otimes \mathcal{F}$. In order to define the interaction Hamiltonian $H_{\mathrm{I}, \kappa}$ of Nelson's model, we use the fact that $\mathcal{H}$ is unitarily equivalent to the constant fiber direct integral $L^{2}\left(\mathbb{R}^{3}, d^{3} x ; \mathcal{F}\right)$, i.e.,

$$
\mathcal{H} \equiv L^{2}\left(\mathbb{R}^{3}\right) \otimes \mathcal{F} \cong L^{2}\left(\mathbb{R}^{3}, d^{3} x ; \mathcal{F}\right) \equiv \int_{\mathbb{R}^{3}}^{\oplus} \mathcal{F} d^{3} x,
$$

see $[3, \S 13]$. Throughout this paper, we identify $\mathcal{H}$ with the constant fiber direct integral, i.e.,

$$
\mathcal{H}=\int_{\mathbb{R}^{3}}^{\oplus} \mathcal{F} d^{3} x
$$

We set

$$
\lambda_{\kappa, x}(k):=\frac{\chi_{\kappa}(k)}{\sqrt{2 \omega(k)}} e^{-i k x}, \quad \forall k, x \in \mathbb{R}^{3} ; \forall \kappa \geq 0
$$

where $\chi_{\kappa}(k):=(2 \pi)^{-3 / 2}$ if $\kappa \leq|k| \leq \Lambda ;:=0$ if $|k|<\kappa$ or $\Lambda<|k|$ for positive constants $\kappa$ and $\Lambda$. Physically, $\kappa$ and $\Lambda$ mean an infrared cutoff and an ultraviolet cutoff, respectively. We fix $\Lambda$ in this paper. Then, we can define $H_{\mathrm{I}, \kappa}$ by

$$
H_{\mathrm{I}, \kappa}:=\int_{\mathbb{R}^{3}}^{\oplus} \phi_{\kappa}(x) d^{3} x,
$$

where $\phi_{\kappa}(x)$ is the cutoff Bose field given by

$$
\phi_{\kappa}(x)=a^{\dagger}\left(\lambda_{\kappa, x}\right)+a\left(\lambda_{\kappa, x}\right) .
$$


We symbolically denote $H_{\mathrm{I}, \kappa}$ by

$$
H_{\mathrm{I}, \kappa}=\int_{\mathbb{R}^{3}} \frac{\chi_{\kappa}(k)}{\sqrt{2 \omega(k)}}\left(e^{i k x} a(k)+e^{-i k x} a^{\dagger}(k)\right) d^{3} k .
$$

It is well known that $H_{\mathrm{I}, \kappa}$ is a self-adjoint operator acting in $\mathcal{H}[3$, Theorem $13-5]$.

From now on, we also denote the identity operator on all Hilbert spaces by $I$. So, for example, $I \otimes I$ is abbreviated to $I$. Moreover, a constant operator with the form of $c I$ is abbreviated to $c$ for a constant $c$.

The cutoff Nelson Hamiltonian is given by

$$
H_{\kappa}^{\mathrm{N}}:=H_{\mathrm{at}} \otimes I+I \otimes H_{\mathrm{f}}+q H_{\mathrm{I}, \kappa}, \quad 0 \leq \forall \kappa<\Lambda ; \forall q \in \mathbb{R},
$$

acting in $\mathcal{H} \equiv L^{2}\left(\mathbb{R}^{3}\right) \otimes \mathcal{F}$. If the infimum of the spectrum of $H_{\kappa}^{\mathrm{N}}$ exists, we call it the ground state energy of $H_{\kappa}^{\mathrm{N}}$. Namely, the ground state energy $E_{\kappa}^{\mathrm{N}}$ of $H_{\kappa}^{\mathrm{N}}$ is defined by

$$
E_{\kappa}^{\mathrm{N}}:=\inf \sigma\left(H_{\kappa}^{\mathrm{N}}\right) .
$$

We say that $H_{\kappa}^{\mathrm{N}}$ has a ground state if $E_{\kappa}^{\mathrm{N}}$ is an eigenvalue of $H_{\kappa}^{\mathrm{N}}$. In this case, every eigenvector with the eigenvalue $E_{\kappa}^{\mathrm{N}}$ is called a ground state. Namely, the ground state $\psi_{\kappa}$ satisfies $H_{\kappa}^{\mathrm{N}} \psi_{\kappa}=E_{\kappa}^{\mathrm{N}} \psi_{\kappa}$. The boson in the ground state $\psi_{\kappa}$ is called soft boson in this paper. We set

$$
H_{\mathrm{N}}:=H_{0}^{\mathrm{N}} \equiv H_{\kappa}^{\mathrm{N}}\left\lceil_{\kappa=0}\right.
$$

and denote the ground state energy of $H_{0}^{\mathrm{N}}$ and $H_{\mathrm{N}}$ by $E_{0}^{\mathrm{N}}$ and $E_{\mathrm{N}}$, respectively, i.e.,

$$
E_{\mathrm{N}}:=\inf \sigma\left(H_{\mathrm{N}}\right)
$$

Then, we have

$$
E_{\kappa}^{\mathrm{N}} \leq\left\langle\psi_{\text {at }} \otimes \Omega_{0}, H_{\kappa}^{\mathrm{N}} \psi_{\text {at }} \otimes \Omega_{0}\right\rangle_{\mathcal{H}}=E_{\text {at }},
$$

where $\langle,\rangle_{\mathcal{H}}$ is the standard inner product of $\mathcal{H}$. We define a non-negative Hamiltonian by

$$
H_{0}:=\left(H_{\mathrm{at}}-E_{\mathrm{at}}\right) \otimes I+I \otimes H_{\mathrm{f}} .
$$

Then, there exist $C_{\Lambda}^{(1)}, C_{\Lambda}^{(2)}>0$ such that

$$
\left\|H_{\mathrm{I}, \kappa} \psi\right\|_{\mathcal{H}} \leq C_{\Lambda}^{(1)}\left\|\left(H_{0}+I\right) \psi\right\|_{\mathcal{H}}+C_{\Lambda}^{(2)}\|\psi\|_{\mathcal{H}}
$$

for every $\psi \in D\left(H_{0}\right)$, which is proved in (6.5) below. Combining this with a Kato-Rellich type argument and the variational characterization of eigenvalues (see, e.g., [3, Theorems 13-10 \& 13-23]), we obtain the following proposition immediately: 
Proposition 2.2. $\quad H_{\kappa}^{\mathrm{N}}, 0 \leq \kappa \leq \Lambda$, is self-adjoint with $D\left(H_{\kappa}^{\mathrm{N}}\right)=D\left(H_{0}\right)$ $\equiv D\left(H_{\text {at }} \otimes I\right) \cap D\left(I \otimes H_{\mathrm{f}}\right) . H_{\kappa}^{\mathrm{N}}, 0 \leq \kappa \leq \Lambda$, is bounded from below for arbitrary values of $q$. In particular,

$$
E_{\text {at }}-q^{2}\left\|\lambda_{\kappa, 0}\right\|_{L^{2}}^{2} \leq E_{\kappa}^{\mathrm{N}} \leq E_{\text {at }} .
$$

Moreover, $H_{\kappa}^{\mathrm{N}}, 0 \leq \kappa \leq \Lambda$, is essentially self-adjoint on every core for $H_{0}$.

It follows from $\omega(k)=|k|$ that in the case $\kappa=0$ Nelson's Hamiltonian $H_{\mathrm{N}} \equiv H_{0}^{\mathrm{N}}=H_{\kappa}^{\mathrm{N}}\left\lceil_{\kappa=0}\right.$ has the singularity at $k=0$ such that

$$
\lim _{|k| \rightarrow 0} \frac{\lambda_{0, x}(k)}{\omega(k)}=\infty \quad \text { and } \quad \frac{\lambda_{0, x}}{\omega} \notin L^{2}\left(\mathbb{R}^{3}\right) .
$$

On the other hand, we have $\lambda_{\kappa, x} / \omega \in L^{2}\left(\mathbb{R}^{3}\right)$ in the case $\kappa>0$. The former condition is called infrared singularity (IRS) condition in [5] (see also [6, (3.5)]), the latter infrared regularity condition.

Denote the number operator of bosons by $N_{\mathrm{f}}$, which is defined as the second quantization of the identity operator $I$, i.e.,

$$
N_{\mathrm{f}}:=d \Gamma(I) .
$$

Symbolically,

$$
N_{\mathrm{f}}=\int_{\mathbb{R}^{3}} a^{\dagger}(k) a(k) d^{3} k .
$$

In [6, Theorem 3.2] the absence theorem is described in terms of the total number of soft bosons forming the cloud in which the Schrödinger particle is dressed. Namely, the statement was that ground state is absent from $D(I \otimes$ $N_{\mathrm{f}}^{1 / 2}$ ). Our theorem is characterized by the spatial localization of the ground state. Namely,

Theorem 2.1 (absence of ground states from $D\left(x^{2} \otimes I\right)$ for $\left.\kappa=0\right)$. Assume (A). For every $q$ with $q \neq 0, H_{\mathrm{N}}=H_{0}^{\mathrm{N}}$ has no ground state in $D\left(x^{2} \otimes I\right)$.

This theorem indirectly says that uncertainty of the position in ground state is infinite. Namely, for the ground state $\psi_{\kappa}$ with $\left\|\psi_{\kappa}\right\|_{\mathcal{H}}=1$ we have symbolically

$$
(\Delta x)_{\mathrm{gs}}:=\left\langle\psi_{\kappa},\left(x \otimes I-\langle x\rangle_{\mathrm{gs}}\right)^{2} \psi_{\kappa}\right\rangle_{\mathcal{H}}^{1 / 2}=\infty,
$$

where $\langle x\rangle_{\mathrm{gs}}$ is the expectation vector of the position in the ground state,

$$
\langle x\rangle_{\mathrm{gs}}:=\left\langle\psi_{\kappa}, x \otimes I \psi_{\kappa}\right\rangle_{\mathcal{H}} \in \mathbb{R}^{3} .
$$


Theorem 2.2 (non-existence of any ground state for $\kappa=0$ ). Let $V$ be in class $(\mathrm{C} 1)$ or $(\mathrm{C} 2)$. Then, for every $q$ with $q \neq 0, H_{\mathrm{N}}=H_{0}^{\mathrm{N}}$ has no ground state in $\mathcal{H}$.

Without loss of generality, we have only to consider a normalized ground state. Thus, we always treat the normalized ground state throughout this paper.

Theorem 2.3 (soft-boson divergence). Assume (A) and that there exists a constant $q_{0}$ such that $H_{\kappa}^{\mathrm{N}}$ has a (normalized) ground state $\psi_{\kappa}$ for every $\kappa$ with $0<\kappa<\Lambda$ and $q$ with $|q|<q_{0}$. If $\psi_{\kappa} \in D\left(x^{2} \otimes I\right)$, then

$$
\begin{aligned}
\left\{\frac{q^{2}}{8 \pi^{2}}\right. & \left.\left(\log \frac{\Lambda}{\kappa}\right)-\frac{q^{2}}{8 \pi^{2}} \Lambda^{2}\left\||x| \otimes I \psi_{\kappa}\right\|_{\mathcal{H}}^{2}\right\} \\
& \leq\left\langle\psi_{\kappa}, I \otimes N_{\mathrm{f}} \psi_{\kappa}\right\rangle_{\mathcal{H}} \\
& \leq\left\{\frac{q^{2}}{2 \pi^{2}}\left(\log \frac{\Lambda}{\kappa}\right)+\frac{q^{2}}{4 \pi^{2}} \Lambda^{2}\left\||x| \otimes I \psi_{\kappa}\right\|_{\mathcal{H}}^{2}\right\} .
\end{aligned}
$$

For the case where $V$ is in class $(\mathrm{C} 2)$, we define a positive constant $q_{\Lambda}$ by

$$
\Sigma-E_{\text {at }}=\frac{q_{\Lambda}^{2}}{4(2 \pi)^{3}} \int_{|k| \leq \Lambda} \frac{|k|}{|k|+k^{2} / 2} d^{3} k,
$$

where $\Sigma:=\inf \sigma_{\text {ess }}\left(H_{\text {at }}\right)$. We set $q_{\Lambda}=\infty$ for the case where $V$ is in class (C1) because $\Sigma=\infty$ in this case. Note that $q_{\Lambda}$ is independent of $\kappa$. By $[13$, Proposition III.3] and [31, Theorem 1] and noting

$$
\frac{\Sigma-E_{\text {at }}}{\frac{1}{2} \int_{\mathbb{R}^{3}}\left|\lambda_{\kappa, x}(k)\right|^{2} k^{2}\left(\omega(k)+k^{2} / 2\right)^{-1} d^{3} k} \geq q_{\Lambda}^{2},
$$

we have the following proposition.

Proposition 2.3. Let us fix $\Lambda>0$. $H_{\kappa}^{\mathrm{N}}$ has a unique ground state $\psi_{\kappa}$ for every $\kappa, q$ with $0<\kappa<\Lambda$ and $|q|<q_{\Lambda}$, provided that $V$ is in class $(\mathrm{C} 1)$ or (C2).

For these ground states $\psi_{\kappa}, 0<\kappa<\Lambda$, we have the following:

Theorem 2.4 (soft-boson divergence). Let $V$ be in $(\mathrm{C} 1)$ or $(\mathrm{C} 2)$. Then, for the ground states $\psi_{\kappa}$ of $H_{\kappa}^{\mathrm{N}}, 0<\kappa<\Lambda$, (2.8) holds. Moreover, 
$\sup _{0<\kappa<\Lambda}|||x| \otimes I \psi_{\kappa} \|_{\mathcal{H}}<\infty$ and

$$
\begin{aligned}
\left\{\frac{q^{2}}{8 \pi^{2}}\right. & \left.\left(\log \frac{\Lambda}{\kappa}\right)-\frac{q^{2}}{8 \pi^{2}} \Lambda^{2} \sup _{0<\kappa<\Lambda}\left\||x| \otimes I \psi_{\kappa}\right\|_{\mathcal{H}}^{2}\right\} \\
& \leq\left\langle\psi_{\kappa}, I \otimes N_{\mathrm{f}} \psi_{\kappa}\right\rangle_{\mathcal{H}} \\
& \leq\left\{\frac{q^{2}}{2 \pi^{2}}\left(\log \frac{\Lambda}{\kappa}\right)+\frac{q^{2}}{4 \pi^{2}} \Lambda^{2} \sup _{0<\kappa<\Lambda}\left\||x| \otimes I \psi_{\kappa}\right\|_{\mathcal{H}}^{2}\right\} .
\end{aligned}
$$

We prove Theorem 2.1 and Theorem 2.3 in $\S 4$ and $\S 5$, respectively. Combining these theorems with the fact on uncertainty argued in $\S 6$, Theorems 2.2 and 2.4 are also proved in $\S 6$.

\section{§3. An Identity from the Operator-theoretical Pull-through Formula}

Let us fix $0 \leq \kappa<\Lambda$, and we suppose that $H_{\kappa}^{\mathrm{N}}$ has a ground state $\psi_{\kappa}$ throughout this section. As declared before Theorem 2.3, for simplicity we normalized $\psi_{\kappa}$ throughout. By using the kernel version of CCR, $\left[a(k), a^{\dagger}\left(k^{\prime}\right)\right]=$ $\delta\left(k-k^{\prime}\right)$, we symbolically obtain the pull-through formula on the ground state $\psi_{\kappa}$,

$$
I \otimes a(k) \psi_{\kappa}=-q \frac{\chi_{\kappa}(k)}{\sqrt{2 \omega(k)}}\left(H_{\kappa}^{\mathrm{N}}-E_{\kappa}^{\mathrm{N}}+\omega(k)\right)^{-1} e^{-i k x} \otimes I \psi_{\kappa} .
$$

However, since the domain of $a(k)$ is so narrow that $a(k)$ is not closable as remarked in Remark 1, (3.1) itself should not be regarded as an operator equality on ground states. It should be regarded as an equality on $L_{\text {loc }}^{2}\left(\mathbb{R}^{3} ; \mathcal{H}\right)$ as Dereziński and Gérard did in [11, Theorem 2.5]. The purposes of this section is to prove the operator-theoretical pull-through formula on the ground state and derive a useful decomposition for Nelson's model from it. To author's best knowledge, the proof in this paper is the first for the pull-through formula in operator theory and the operator-theoretical version of this formula has another development in operator theory of IR catastrophe (cf. [7, 19, 21]).

Before we state our desired proposition, we note the following lemma.

Lemma 3.1. For $f \in L^{2}\left(\mathbb{R}^{3}\right)$ and $t \in \mathbb{R}$, set

$$
a_{t}(f):=e^{i t H_{\kappa}^{N}}\left(I \otimes a\left(e^{-i \omega t} f\right)\right) e^{-i t H_{\kappa}^{N}} .
$$

If $\omega f, f / \sqrt{\omega} \in L^{2}\left(\mathbb{R}^{3}\right)$, then

$$
\frac{d}{d t} a_{t}(f) \psi=-i q e^{i t H_{\kappa}^{N}}\left\{\left(\int_{\mathbb{R}^{3}} f(k)^{*} e^{i t \omega(k)} \lambda_{\kappa, x}(k) d^{3} k\right) \otimes I\right\} e^{-i t H_{\kappa}^{N}} \psi
$$

for every $\psi \in D\left(\left(H_{\kappa}^{N}\right)^{2}\right)$. 
Proof. In the same way as in [23, Theorem 4.1], we can prove that

$$
\frac{d}{d t} a_{t}(f) \psi=i e^{i t H_{\kappa}^{\mathrm{N}}}\left[q H_{\mathrm{I}, \kappa}, I \otimes a\left(e^{-i \omega t} f\right)\right] e^{-i t H_{\kappa}^{\mathrm{N}}} \psi
$$

for every $\psi \in D\left(\left(H_{\kappa}^{\mathrm{N}}\right)^{2}\right)$. We obtain (3.2) from this equation directly.

Proposition 3.1 (pull-through formula on ground states). Fix $\kappa$ with $0 \leq \kappa<\Lambda$. Assume $(\mathrm{A})$ and suppose that $H_{\kappa}^{\mathrm{N}}$ has a ground state $\psi_{\kappa}$. If $\psi_{\kappa} \in D\left(x^{2} \otimes I\right)$, then for all $f \in C_{0}^{\infty}\left(\mathbb{R}^{3} \backslash\{0\}\right)$,

$$
\begin{aligned}
I & \otimes a(f) \psi_{\kappa} \\
& =-q \int_{\mathbb{R}^{3}} f(k)^{*} \frac{\chi_{\kappa}(k)}{\sqrt{2 \omega(k)}}\left(H_{\kappa}^{\mathrm{N}}-E_{\kappa}^{\mathrm{N}}+\omega(k)\right)^{-1}\left(e^{-i k x} \otimes I\right) \psi_{\kappa} d^{3} k .
\end{aligned}
$$

Proof. Let $f \in C_{0}^{\infty}\left(\mathbb{R}^{3} \backslash\{0\}\right)$. Then, there exists $d_{f}>0$ such that $\left\{k \in \mathbb{R}^{3}|| k \mid<d_{f}\right\} \subset \mathbb{R}^{3} \backslash \operatorname{supp} f$, which implies supp $f \subset\left\{k \in \mathbb{R}^{3}|| k \mid>d_{f} / 2\right\}$. Set $\Omega_{\kappa, \Lambda}^{\text {int }}:=\left\{k \in \mathbb{R}^{3}|\kappa<| k \mid<\Lambda\right\}$ and $\Omega_{\kappa, \Lambda}^{\text {ext }}:=\left\{k \in \mathbb{R}^{3}|0<| k \mid<\kappa\right.$ or $\Lambda<|k|\}$. Since $L^{2}\left(\mathbb{R}^{3}\right) \cong L^{2}\left(\Omega_{\kappa, \Lambda}^{\text {int }}\right) \oplus L^{2}\left(\Omega_{\kappa, \Lambda}^{\text {ext }}\right)$, we identify $L^{2}\left(\mathbb{R}^{3}\right)$ with $L^{2}\left(\Omega_{\kappa, \Lambda}^{\text {int }}\right) \oplus L^{2}\left(\Omega_{\kappa, \Lambda}^{\text {ext }}\right)$ in this proof. There exists $f^{\sharp} \in L^{2}\left(\Omega_{\kappa, \Lambda}^{\sharp}\right), \sharp=$ int, ext, such that $L^{2}\left(\mathbb{R}^{3}\right) \ni f=f^{\text {int }} \oplus f^{\text {ext }} \in L^{2}\left(\Omega_{\kappa, \Lambda}^{\text {int }}\right) \oplus L^{2}\left(\Omega_{\kappa, \Lambda}^{\text {ext }}\right)$. For $f^{\sharp}$, there exists a sequence $f_{\nu}^{\sharp} \in C_{0}^{\infty}\left(\Omega_{\kappa, \Lambda}^{\sharp}\right), \nu \in \mathbb{N}$, such that $f_{\nu}^{\sharp} \rightarrow f^{\sharp}$ in $L^{2}\left(\Omega_{\kappa, \Lambda}^{\sharp}\right)$ as $\nu \rightarrow \infty$ and $\operatorname{supp}\left(f_{\nu}^{\text {int }} \oplus f_{\nu}^{\text {ext }}\right) \subset\left\{k \in \mathbb{R}^{3}|| k \mid>d_{f} / 2\right\}$ for each $\nu$. For simplicity, we denote $f_{\nu}^{\text {int }} \oplus f_{\nu}^{\text {ext }}$ by $f_{\nu}$, i.e., $f_{\nu}:=f_{\nu}^{\text {int }} \oplus f_{\nu}^{\text {ext }}$.

For every $\psi \in D\left(\left(H_{\kappa}^{\mathrm{N}}\right)^{2}\right), t \in \mathbb{R}$, and the above $f_{\nu}$, we have

$$
\begin{aligned}
a_{t}\left(f_{\nu}\right) \psi= & I \otimes a\left(f_{\nu}\right) \psi \\
& -i q \int_{0}^{t} e^{i s H_{\kappa}^{\mathrm{N}}}\left\{\left(\int_{\mathbb{R}^{3}} f_{\nu}(k)^{*} e^{i s \omega(k)} \lambda_{\kappa, x}(k) d^{3} k\right) \otimes I\right\} e^{-i s H_{\kappa}^{\mathrm{N}}} \psi d s
\end{aligned}
$$

by Lemma 3.1. Here, we note that $\operatorname{supp}\left(f_{\nu}^{*} \lambda_{\kappa, x}\right)=\operatorname{supp}\left(\left(f_{\nu}^{\text {int }}\right)^{*} \lambda_{\kappa, x}\right)$. Since $f_{\nu}^{*} \lambda_{\kappa, x} \in C_{0}^{\infty}\left(\Omega_{\kappa, \Lambda}^{\text {int }}\right)$, we obtain by partial integration as in [6, Lemma 4.3$]$ that

$$
\int_{\mathbb{R}^{3}} f_{\nu}(k)^{*} e^{i t \omega(k)} \lambda_{\kappa, x}(k) d^{3} k=-\frac{1}{t^{2}} \int_{\mathbb{R}^{3}} g(k) e^{i t \omega(k)} d^{3} k,
$$

where for $n, m=1,2,3$,

$$
\begin{aligned}
g(k) & =\partial_{m}\left\{\frac{1}{\partial_{m} \omega(k)} \partial_{n}\left(\frac{1}{\partial_{n} \omega(k)} \lambda_{\kappa, x}(k) f_{\nu}(k)^{*}\right)\right\} \\
& =\partial_{m}\left\{\frac{1}{\partial_{m} \omega(k)} \partial_{n}\left(e^{-i k x} \frac{1}{\partial_{n} \omega(k)} \lambda_{\kappa, 0}(k) f_{\nu}(k)^{*}\right)\right\}
\end{aligned}
$$


with $\partial_{n}:=\partial / \partial k_{n}$. Concerning $\partial_{n} \lambda_{\kappa, x}$ and $\partial_{m} \partial_{n} \lambda_{\kappa, x}$ in the above expression of $g(k)$, we can directly estimate them in the following because the function of $x$ appearing in $\lambda_{\kappa, x}$ is only $e^{-i k x}$. There exists $C_{\Lambda, \nu}>0$, which is independent of $\kappa, x$, such that $\left|\partial_{n} \lambda_{\kappa, x}(k)\right| \leq C_{\Lambda, \nu}(1+|x|)$ and $\left|\partial_{m} \partial_{n} \lambda_{\kappa, x}(k)\right| \leq C_{\Lambda, \nu}\left(1+|x|^{2}\right)$ for every $k$ with $\kappa<|k|<\Lambda$. Thus, we have $g \in L\left(\mathbb{R}^{3}\right)$ and we can show that $a_{ \pm}\left(f_{\nu}\right) \psi=s-\lim _{t \rightarrow \pm \infty} a_{t}\left(f_{\nu}\right) \psi$ exists for all $\psi \in D\left(H_{\kappa}^{\mathrm{N}^{2}}\right) \cap D\left(x^{2} \otimes I\right)$ in the same way as in [6, Lemma 4.3]. So, we have the following equality

$$
\begin{aligned}
a_{ \pm}\left(f_{\nu}\right) \psi= & I \otimes a\left(f_{\nu}\right) \psi \\
& -i q \int_{0}^{ \pm \infty} e^{i t H_{\kappa}^{\mathrm{N}}}\left\{\left(\int_{\mathbb{R}^{3}} f_{\nu}(k)^{*} e^{i t \omega(k)} \lambda_{\kappa, x}(k) d^{3} k\right) \otimes I\right\} e^{-i t H_{\kappa}^{\mathrm{N}}} \psi d t .
\end{aligned}
$$

Also see [22, Theorem 1 and (6)] and [23, Theorem 5.1]. Moreover, using the absolute continuity of $\omega(k)$ and the Riemann-Lebesgue theorem, we have $a_{ \pm}\left(f_{\nu}\right) \psi_{\kappa}=0$. By using these facts and $e^{-i t H_{\kappa}^{\mathrm{N}}} \psi_{\kappa}=e^{-i t E_{\kappa}^{\mathrm{N}}} \psi_{\kappa}$, we have

$$
\begin{aligned}
I & \otimes \\
& a\left(f_{\nu}\right) \psi_{\kappa} \\
& =i q \int_{0}^{\infty} e^{i t\left(H_{\kappa}^{\mathrm{N}}-E_{\kappa}^{\mathrm{N}}\right)}\left(\int_{\mathbb{R}^{3}} f_{\nu}(k)^{*} e^{i t \omega(k)} \lambda_{\kappa, x}(k) d^{3} k\right) \otimes I \psi_{\kappa} d t .
\end{aligned}
$$

So, by Fubini's theorem and Lebesgue's dominated convergence theorem, we have for every $\phi \in D\left(H_{\kappa}^{\mathrm{N}}\right)$

$$
\begin{aligned}
\langle\phi, I & \left.\otimes a\left(f_{\nu}\right) \psi_{\kappa}\right\rangle_{\mathcal{H}} \\
& =i q \lim _{\varepsilon \downarrow 0} \int_{0}^{\infty} e^{-t \varepsilon}\left(\int_{\mathbb{R}^{3}} f_{\nu}(k)^{*}\left\langle\phi, e^{i t\left(H_{\kappa}^{\mathrm{N}}-E_{\kappa}^{\mathrm{N}}+\omega(k)\right)} \lambda_{\kappa, x} \otimes I \psi_{\kappa}\right\rangle_{\mathcal{H}} d^{3} k\right) d t \\
& =i q \lim _{\varepsilon \downarrow 0} \int_{\mathbb{R}^{3}} f_{\nu}(k)^{*}\left\langle\int_{0}^{\infty} e^{-i t\left(H_{\kappa}^{\mathrm{N}}-E_{\kappa}^{\mathrm{N}}+\omega(k)-i \varepsilon\right)} \phi d t, \lambda_{\kappa, x} \otimes I \psi_{\kappa}\right\rangle_{\mathcal{H}} d^{3} k \\
& =i q \lim _{\varepsilon \downarrow 0} \int_{\mathbb{R}^{3}} f_{\nu}(k)^{*}\left\langle-i\left(H_{\kappa}^{\mathrm{N}}-E_{\kappa}^{\mathrm{N}}+\omega(k)-i \varepsilon\right)^{-1} \phi, \lambda_{\kappa, x} \otimes I \psi_{\kappa}\right\rangle_{\mathcal{H}} d^{3} k \\
& =\left\langle\phi,-q \int_{\mathbb{R}^{3}} f_{\nu}(k)^{*}\left(H_{\kappa}^{\mathrm{N}}-E_{\kappa}^{\mathrm{N}}+\omega(k)\right)^{-1} \lambda_{\kappa, x} \otimes I \psi_{\kappa} d^{3} k\right\rangle_{\mathcal{H}},
\end{aligned}
$$

where we used Fubini's theorem in the 2nd equality noting

$$
\begin{aligned}
& \left|e^{-t \varepsilon} f_{\nu}(k)^{*}\left\langle e^{-i t\left(H_{\kappa}^{\mathrm{N}}-E_{\kappa}^{\mathrm{N}}+\omega(k)\right)} \phi, \lambda_{\kappa, x} \otimes I \psi_{\kappa}\right\rangle_{\mathcal{H}}\right| \\
& \leq e^{-t \varepsilon}\left|f_{\nu}(k)\right|\left|\lambda_{\kappa, x}(k)\right|\|\phi\|_{\mathcal{H}},
\end{aligned}
$$


and we calculated the integral over $0<t<\infty$ in the 3rd equality using

$$
\begin{aligned}
& \lim _{T \rightarrow \infty} i\left(H_{\kappa}^{\mathrm{N}}-E_{\kappa}^{\mathrm{N}}+\omega(k)-i \varepsilon\right)^{-1} e^{-i T\left(H_{\kappa}^{\mathrm{N}}-E_{\kappa}^{\mathrm{N}}+\omega(k)-i \varepsilon\right)} \phi \\
& \quad=\lim _{T \rightarrow \infty} e^{-T \varepsilon} i\left(H_{\kappa}^{\mathrm{N}}-E_{\kappa}^{\mathrm{N}}+\omega(k)-i \varepsilon\right)^{-1} e^{-i T\left(H_{\kappa}^{\mathrm{N}}-E_{\kappa}^{\mathrm{N}}+\omega(k)\right)} \phi=0 .
\end{aligned}
$$

Therefore, (3.3) for $f_{\nu}$ follows from (3.4).

If $k \in \operatorname{supp} f \cup\left(\bigcup_{\nu \geq \nu_{0}} \operatorname{supp} f_{\nu}\right)$, then $|k|^{-1}<2 / d_{f}$. Hence it follows that $\left\|f_{\nu} / \sqrt{\omega}-f / \sqrt{\omega}\right\|_{L^{2}}^{2} \leq 2 d_{f}^{-1}\left\|f_{\nu}-f\right\|_{L^{2}}^{2}=2 d_{f}^{-1}\left(\left\|f_{\nu}^{\mathrm{int}}-f^{\mathrm{int}}\right\|_{L^{2}\left(\Omega_{\kappa, \Lambda}^{\mathrm{int}}\right)}^{2}+\| f_{\nu}^{\mathrm{ext}}-\right.$ $\left.f^{\text {ext }} \|_{L^{2}\left(\Omega_{\kappa, \Lambda}^{\text {ext }}\right)}^{2}\right)$ for $\nu \geq \nu_{0}$. Therefore, we obtain

$$
f_{\nu} / \omega^{j / 2} \longrightarrow f / \omega^{j / 2}
$$

in $L^{2}\left(\mathbb{R}^{3}\right)$ as $\nu \rightarrow \infty$ for $j=0,1$. Since $\psi_{\kappa} \in D\left(H_{0}^{1 / 2}\right)$ by Proposition 2.2 , the fundamental inequality $\left\|I \otimes a\left(f_{\nu}\right) \psi_{\kappa}-I \otimes a(f) \psi_{\kappa}\right\|_{\mathcal{H}} \leq\left\|\left(f_{\nu}-f\right) / \sqrt{\omega}\right\|_{L^{2}} \| I \otimes$ $H_{0}^{1 / 2} \psi_{\kappa} \|_{\mathcal{H}}$ holds. So, by $(3.5), I \otimes a\left(f_{\nu}\right) \psi_{\kappa} \longrightarrow I \otimes a(f) \psi_{\kappa}$ as $\nu \rightarrow \infty$. By the Schwarz inequality, $\chi_{0} \omega^{-1} \in L^{2}\left(\mathbb{R}^{3}\right)$, and (3.5), the r.h.s of (3.3) for $f_{\nu}$ converges to that for $f$. Therefore, (3.3) holds for $f \in C_{0}^{\infty}\left(\mathbb{R}^{3} \backslash\{0\}\right)$.

In (3.1), we employ the following decomposition of the plain wave $e^{-i k x}$ into the dipole-approximated term $e^{-i k 0}=1$ and the error term $e^{-i k x}-1$, i.e.,

$$
e^{-i k x}=1+\left(e^{-i k x}-1\right)
$$

because this decomposition provides very simple treatment to estimate the total number of soft bosons. Dereziński and Gérard implement this way in $L^{2}$-theory [11]. We also employ this way and implement it in operator theory by using (3.3).

Proposition 3.2. Let us fix $\kappa$ with $0 \leq \kappa<\Lambda$, and suppose that $H_{\kappa}^{\mathrm{N}}$ has a ground state $\psi_{\kappa}$ and $\psi_{\kappa} \in D\left(x^{2} \otimes I\right)$. Then, for all $f \in C_{0}^{\infty}\left(\mathbb{R}^{3} \backslash\{0\}\right)$,

$$
I \otimes a(f) \psi_{\kappa}=\sum_{j=1}^{2} \int_{\mathbb{R}^{3}} f(k)^{*} J_{j}(k) \psi_{\kappa} d^{3} k
$$

with

$$
\begin{aligned}
& J_{1}(k)=-q \frac{\chi_{\kappa}(k)}{\sqrt{2 \omega(k)} \omega(k)} I \otimes I, \\
& J_{2}(k)=-q \frac{\chi_{\kappa}(k)}{\sqrt{2 \omega(k)}}\left(H_{\kappa}^{\mathrm{N}}-E_{\kappa}^{\mathrm{N}}+\omega(k)\right)^{-1}\left(e^{-i k x}-1\right) \otimes I .
\end{aligned}
$$


Then,

$$
\begin{aligned}
& \int_{\mathbb{R}^{3}}\left\|J_{1}(k) \psi_{\kappa}\right\|_{\mathcal{H}}^{2} d^{3} k=\frac{q^{2}}{4 \pi^{2}} \log \frac{\Lambda}{\kappa}, \\
& \int_{\mathbb{R}^{3}}\left\|J_{2}(k) \psi_{\kappa}\right\|_{\mathcal{H}}^{2} d^{3} k \leq \frac{q^{2}}{8 \pi^{2}} \Lambda^{2}\left\||x| \otimes I \psi_{\kappa}\right\|_{\mathcal{H}}^{2} .
\end{aligned}
$$

Proof. We obtain immediately (3.7) from (3.3) by using (3.6) and $\left(H_{\kappa}^{\mathrm{N}}-\right.$ $\left.E_{\kappa}^{\mathrm{N}}+\omega(k)\right)^{-1} \psi_{\kappa}=\omega(k)^{-1} \psi_{\kappa}$. (3.8) follows from a direct computation. By using $\left|e^{-i k x}-1\right| \leq|k||x|$, we have (3.9).

Remark 2. We note that decomposition (3.6) is not always useful in proving the non-existence of ground state. We have to use another technique in a general case (e.g. see GSB model and some polaron models [19]). In fact, to treat several sorts of polarons, we mathematically consider more general dispersion relations $\omega(k)$ and coupling functions $\lambda_{\kappa, x}(k)$. For simplicity, we consider $\omega(k)=|k|^{\mu}$ and $\lambda_{\kappa, x}(k)=\chi_{\kappa}(k)|k|^{-\nu} e^{-i k x}$ now, where $\mu \geq 0, \nu \in \mathbb{R}$, and $d=1,2,3$. Then, because we do not always have (3.9), our argument in $\S 4$ does not work. For example, consider the case $\mu+2 \nu<d \leq 2 \mu+2 \nu-2$. For such a case, by following the idea in [6] instead of (3.6), we can press forward with a concrete computation from [6, Lemma 5.1] as announced in [18]. For further details, see [19].

\section{$\S 4$. Absence of Ground State from $D\left(x^{2} \otimes I\right)$ for $\kappa=0$}

In [6] we proved that any ground state of GSB model is absent from $D(I \otimes$ $N_{\mathrm{f}}^{1 / 2}$ ). Here, by employing decomposition (3.7), we prove Theorem 2.1, namely, any ground state of $H_{\mathrm{N}}=H_{0}^{\mathrm{N}}$ is absent from $D\left(x^{2} \otimes I\right)$.

Proof of Theorem 2.1: We use reductio ad absurdum to prove Theorem 2.1. Suppose that $H_{\mathrm{N}}:=H_{0}^{\mathrm{N}}$ has a ground state $\psi_{0}$ in $D\left(x^{2} \otimes I\right)$. We note we already normalized the ground state $\psi_{0}$. For every $\phi \in D\left(I \otimes N_{\mathrm{f}}^{1 / 2}\right)$, define the function $F_{\phi, \psi_{0}}$ by

$$
F_{\phi, \psi_{0}}(k)=\sum_{j=1}^{2}\left\langle\phi, J_{j}(k) \psi_{0}\right\rangle_{\mathcal{H}} .
$$

Since $D\left(I \otimes N_{\mathrm{f}}^{1 / 2}\right) \subset D\left(I \otimes a^{\dagger}(f)\right)$, we can define the anti-linear functional $T_{\phi, \psi_{0}}: L^{2}\left(\mathbb{R}^{3}\right) \rightarrow \mathbb{C}$ by

$$
T_{\phi, \psi_{0}}(f)=\left\langle I \otimes a^{\dagger}(f) \phi, \psi_{0}\right\rangle_{\mathcal{H}}, \quad \forall \phi \in D\left(I \otimes N_{\mathrm{f}}^{1 / 2}\right) .
$$


By the fundamental inequality concerning $a^{\dagger}(f)$ and $N_{\mathrm{f}}$, we have

$$
\left|T_{\phi, \psi_{0}}(f)\right| \leq\left\|I \otimes\left(N_{\mathrm{f}}+1\right)^{1 / 2} \phi\right\|_{\mathcal{H}}\|f\|_{L^{2}},
$$

namely, $T_{\phi, \psi_{0}}$ is a bounded anti-linear functional. So, by Riesz's lemma, there exists a unique $F \in L^{2}\left(\mathbb{R}^{3}\right)$ such that $T_{\phi, \psi_{0}}(f)=\langle f, F\rangle_{L^{2}}$ for every $f \in$ $L^{2}\left(\mathbb{R}^{3}\right)$. We note that $\psi_{0} \in D\left(H_{\mathrm{N}}\right)=D\left(H_{0}\right) \subset D\left(H_{0}^{1 / 2}\right) \subset D\left(H_{\mathrm{f}}^{1 / 2}\right) \subset D(a(g))$ for every $g \in L^{2}\left(\mathbb{R}^{3}\right)$ with $g / \sqrt{\omega} \in L^{2}\left(\mathbb{R}^{3}\right)$. By $(3.7)$, we obtain $\left\langle f, F_{\phi, \psi_{0}}\right\rangle_{L^{2}}=$ $\left\langle\phi, I \otimes a(f) \psi_{0}\right\rangle_{\mathcal{H}}=T_{\phi, \psi_{0}}(f)$ for $f \in C_{0}^{\infty}\left(\mathbb{R}^{3} \backslash\{0\}\right)$. Thus, we have

$$
F_{\phi, \psi_{0}}=F \in L^{2}\left(\mathbb{R}^{3}\right), \quad \forall \phi \in D\left(I \otimes N_{\mathrm{f}}^{1 / 2}\right) .
$$

By (3.7) and (4.1), we have

$$
-q \Theta_{1}(k)\left\langle\phi, \psi_{0}\right\rangle_{\mathcal{H}}=\left\langle\phi, J_{1}(k) \psi_{0}\right\rangle_{\mathcal{H}}=F_{\phi, \psi_{0}}(k)-\left\langle\phi, J_{2}(k) \psi_{0}\right\rangle_{\mathcal{H}}
$$

as an $L^{2}\left(\mathbb{R}^{3}\right)$-function of $k$, where

$$
\Theta_{1}(k)=\frac{\chi_{0}(k)}{\sqrt{2 \omega(k)} \omega(k)} .
$$

So, by (3.8) and (3.9), we reach a contradiction if $\left\langle\phi, \psi_{0}\right\rangle_{\mathcal{H}} \neq 0$. Namely, the left hand side of $(4.2)$ is not in $L^{2}\left(\mathbb{R}^{3}\right)$ when $\left\langle\phi, \psi_{0}\right\rangle_{\mathcal{H}} \neq 0$, on the other hand, the right hand side of $(4.2)$ is in $L^{2}\left(\mathbb{R}^{3}\right)$. Let us consider the case where $\left\langle\phi, \psi_{0}\right\rangle_{\mathcal{H}}=0$ now. In this case, since we took an arbitrary $\phi$ from $D\left(I \otimes N_{\mathrm{f}}^{1 / 2}\right)$ which is dense in $L^{2}\left(\mathbb{R}^{3}\right)$, we have $\psi_{0}=0$, which also implies a contradiction. Therefore, we obtain Theorem 2.1.

\section{$\S 5$. Sharp Estimate of Total Number of Soft Bosons}

In this section, we prove Theorem 2.3. So, we assume $\kappa>0$ throughout this section. In order to prove Theorem 2.3, we justify the following symbolic identity

$$
\left\langle\psi_{\kappa}, I \otimes N_{\mathrm{f}} \psi_{\kappa}\right\rangle_{\mathcal{H}}=\int_{\mathbb{R}^{3}}\left\|I \otimes a(k) \psi_{\kappa}\right\|_{\mathcal{H}}^{2} d^{3} k .
$$

Let $X=(X, \mathcal{A}, \mu)$ be a $\sigma$-finite measurable space. Define the symmetric Fock space $\mathcal{F}_{X}$ from $X$ by

$$
\mathcal{F}_{X}=\bigoplus_{n=0}^{\infty} \otimes_{s}^{n} L^{2}(X) .
$$

The annihilation operator $a(f), f \in L^{2}(X)$, and the number operator $N$ acting in $\mathcal{F}_{X}$ can be defined in the same way as in (2.1) and (2.6) for those acting in $\mathcal{F}$, respectively. 
Proposition 5.1. For arbitrary complete orthonormal system $\left\{f_{\nu}\right\}_{\nu}$ of $L^{2}(X)$,

$$
\left\|N^{1 / 2} \Psi\right\|_{\mathcal{F}_{X}}^{2}=\sum_{\nu=1}^{\infty}\left\|a\left(f_{\nu}\right) \Psi\right\|_{\mathcal{F}_{X}}^{2}, \quad \forall \Psi \in D\left(N^{1 / 2}\right) .
$$

Proof. Set

$$
\begin{aligned}
\Psi_{M}^{(n)}\left(k_{1}, \cdots, k_{n}\right) & =\sum_{\nu=1}^{M}\left|\left(f_{\nu}, \Psi^{(n+1)}\left(\cdot, k_{1}, \cdots, k_{n}\right)\right)_{L^{2}(X)}\right|^{2}, \\
d \mu^{n}\left(k_{1}, \cdots, k_{n}\right) & =d \mu\left(k_{1}\right) \otimes \cdots \otimes d \mu\left(k_{n}\right) .
\end{aligned}
$$

By the definition of the annihilation operator, for each $M \in \mathbb{N}$ and every $\Psi \in D\left(N^{1 / 2}\right)$ we have

$$
\sum_{\nu=1}^{M}\left\|a\left(f_{\nu}\right) \Psi\right\|_{\mathcal{F}_{X}}^{2}=\sum_{n=0}^{\infty}(n+1) \int_{X^{n}} \Psi_{M}^{(n)}\left(k_{1}, \cdots, k_{n}\right) d \mu^{n}\left(k_{1}, \cdots, k_{n}\right) .
$$

Since $\Psi^{(n+1)}\left(\cdot, k_{1}, \cdots, k_{n}\right) \in L^{2}(X)$ for $\mu^{n}$-a.e. $\left(k_{1}, \cdots, k_{n}\right) \in X^{n}$, we have

$$
\Psi_{M}^{(n)}\left(k_{1}, \cdots, k_{n}\right) \leq\left\|\Psi^{(n+1)}\left(\cdot, k_{1}, \cdots, k_{n}\right)\right\|_{L^{2}(X)}^{2}, \mu^{n} \text {-a.e. }\left(k_{1}, \cdots, k_{n}\right) \in X^{n},
$$

by Bessel's inequality. Since $\left\{f_{\nu}\right\}_{\nu}$ is complete, $\Psi_{M}^{(n)}\left(k_{1}, \cdots, k_{n}\right)$ converges to $\left\|\Psi^{(n+1)}\left(\cdot, k_{1}, \cdots, k_{n}\right)\right\|_{L^{2}(X)}^{2}$ as $M \rightarrow \infty$. Therefore, (5.2) follows from Lebesgue's monotone convergence theorem.

Lemma 5.1. For every $\kappa$ with $0<\kappa<\Lambda$,

$$
\psi_{\kappa} \in D\left(I \otimes N_{\mathrm{f}}\right) .
$$

Proof. Let $\mathbb{R}_{\leq \kappa}^{3}=\left\{k \in \mathbb{R}^{3}|| k \mid \leq \kappa\right\}$ and $\mathbb{R}_{>\kappa}^{3}=\left\{k \in \mathbb{R}^{3}|| k \mid>\kappa\right\}$. We set $N_{\mathrm{f}}^{\leq \kappa}=d \Gamma\left(\mathbb{1}_{[0, \kappa]}\right)$ and $N_{\mathrm{f}}^{>\kappa}=d \Gamma\left(\mathbb{1}_{(\kappa, \infty)}\right)$ acting in $\bigoplus_{n=0}^{\infty} \otimes_{\mathrm{s}}^{n} L^{2}\left(\mathbb{R}_{\leq \kappa}^{3}\right)$ and $\bigoplus_{n=0}^{\infty} \otimes_{\mathrm{s}}^{n} L^{2}\left(\mathbb{R}_{>\kappa}^{3}\right)$, respectively. We note

$$
D\left(\left(H_{\mathrm{f}}^{>\kappa}\right)^{s}\right) \subset D\left(\left(N_{\mathrm{f}}^{>\kappa}\right)^{s}\right)
$$

for $s>0$. Through the unitary equivalence $H_{\kappa}^{\mathrm{N}} \cong H_{\mathrm{f}}^{\leq \kappa} \otimes I+I \otimes H_{\mathrm{N}}^{>\kappa}$, the ground state $\psi_{\kappa}$ of $H_{\kappa}^{\mathrm{N}}$ is represented by $\Omega_{0} \otimes \psi_{\kappa}^{>\kappa}$, where $\psi_{\kappa}^{>\kappa}$ is a ground state of $H_{\mathrm{N}}^{>\kappa}$ and $\Omega_{0}$ the Fock vacuum. We note $N_{\mathrm{f}} \cong N_{\mathrm{f}}^{\leq \kappa} \otimes I+I \otimes N_{\mathrm{f}}^{>\kappa}$. Since $D\left(H_{\mathrm{f}}^{>\kappa}\right) \subset D\left(N_{\mathrm{f}}^{>\kappa}\right)$ by $(5.3), \psi_{\kappa}^{>\kappa}$ is in $D\left(N_{\mathrm{f}}^{>\kappa}\right)$, i.e., $\psi_{\kappa}^{>\kappa} \in D\left(N_{\mathrm{f}}^{>\kappa}\right)$, by Proposition 2.2. Hence our lemma follows.

Setting $X=\mathbb{R}^{3}$ in Proposition 5.1 and using the identification (2.4) and Lemma 5.1, we obtain mathematical justification of (5.1): 
Corollary 5.1. For every $\kappa$ with $0<\kappa<\Lambda$ and an arbitrary complete orthonormal system $\left\{f_{\nu}\right\}_{\nu=1}^{\infty}$ of $L^{2}\left(\mathbb{R}^{3}\right)$,

$$
\left\langle\psi_{\kappa}, I \otimes N_{\mathrm{f}} \psi_{\kappa}\right\rangle_{\mathcal{H}}=\left\|I \otimes N_{\mathrm{f}}^{1 / 2} \psi_{\kappa}\right\|_{\mathcal{H}}^{2}=\sum_{\nu=1}^{\infty}\left\|I \otimes a\left(f_{\nu}\right) \psi_{\kappa}\right\|_{\mathcal{H}}^{2} .
$$

Proof of Theorem 2.3: Fix $\kappa$ satisfying $0<\kappa<\Lambda$. We assume all hypotheses of Theorem 2.3. Let $\left\{f_{\nu}\right\}_{\nu=1}^{\infty} \subset C_{0}^{\infty}\left(\mathbb{R}^{3} \backslash\{0\}\right)$ and $\left\{e_{p}\right\}_{p=1}^{\infty}$ be complete orthonormal systems of $L^{2}\left(\mathbb{R}^{3}\right)$ and $\mathcal{H}$, respectively. Then, $\left\{f_{\nu}(\cdot) e_{p}\right\}_{\nu, p=1}^{\infty}$ is a complete orthonormal system of $L^{2}\left(\mathbb{R}^{3} ; \mathcal{H}\right)$. By using Parseval's equality, we have

$$
\begin{aligned}
\int_{\mathbb{R}^{3}}\left\|J_{j}(k) \psi_{\kappa}\right\|_{\mathcal{H}}^{2} d^{3} k & \equiv\left\|J_{j}(\cdot) \psi_{\kappa}\right\|_{L^{2}\left(\mathbb{R}^{3} ; \mathcal{H}\right)}^{2} \\
& =\sum_{\nu=1}^{\infty} \sum_{p=1}^{\infty}\left|\int_{\mathbb{R}^{3}}\left\langle f_{\nu}(k) e_{p}, J_{j}(k) \psi_{\kappa}\right\rangle_{\mathcal{H}} d^{3} k\right|^{2} \\
& =\sum_{\nu=1}^{\infty} \sum_{p=1}^{\infty}\left|\left\langle e_{p}, \int_{\mathbb{R}^{3}} f_{\nu}(k)^{*} J_{j}(k) \psi_{\kappa} d^{3} k\right\rangle_{\mathcal{H}}\right|^{2} \\
& =\sum_{\nu=1}\left\|\int_{\mathbb{R}^{3}} f_{\nu}(k)^{*} J_{j}(k) \psi_{\kappa} d^{3} k\right\|_{\mathcal{H}}^{2}
\end{aligned}
$$

for $j=1,2$ since $J_{j}(\cdot) \psi_{\kappa} \in L^{2}\left(\mathbb{R}^{3} ; \mathcal{H}\right)$ for every $\kappa$ satisfying $0<\kappa<\Lambda$. Applying the triangle inequality to (3.7) and using (5.4) and (5.5), we have

$$
\begin{aligned}
\left\langle\psi_{\kappa}, I \otimes N_{\mathrm{f}} \psi_{\kappa}\right\rangle_{\mathcal{H}} & =\sum_{\nu=1}^{\infty}\left\|I \otimes a\left(f_{\nu}\right) \psi_{\kappa}\right\|_{\mathcal{H}}^{2} \\
& \leq 2 \int_{\mathbb{R}^{3}}\left\|J_{1}(k) \psi_{\kappa}\right\|_{\mathcal{H}}^{2}+2 \int_{\mathbb{R}^{3}}\left\|J_{2}(k) \psi_{\kappa}\right\|_{\mathcal{H}}^{2} .
\end{aligned}
$$

By (3.8), (3.9), (5.6), we have

$$
\left\langle\psi_{\kappa}, I \otimes N_{\mathrm{f}} \psi_{\kappa}\right\rangle_{\mathcal{H}} \leq 2\left\{\frac{q^{2}}{4 \pi^{2}}\left(\log \frac{\Lambda}{\kappa}\right)+\frac{q^{2}}{8 \pi^{2}} \Lambda^{2}|||x| \otimes I \psi_{\kappa} \|_{\mathcal{H}}^{2}\right\} .
$$

By (3.7) again, we have

$$
\int_{\mathbb{R}^{3}} f_{\nu}(k)^{*} J_{1}(k) \psi_{\kappa} d^{3} k=I \otimes a\left(f_{\nu}\right) \psi_{\kappa}-\int_{\mathbb{R}^{3}} f_{\nu}(k)^{*} J_{2}(k) \psi_{\kappa} d^{3} k .
$$

In the same way as above, we get

$$
\int_{\mathbb{R}^{3}}\left\|J_{1}(k) \psi_{\kappa}\right\|_{\mathcal{H}}^{2} d^{3} k \leq 2\left\langle\psi_{\kappa}, I \otimes N_{\mathrm{f}} \psi_{\kappa}\right\rangle_{\mathcal{H}}+2 \int_{\mathbb{R}^{3}}\left\|J_{2}(k) \psi_{\kappa}\right\|_{\mathcal{H}}^{2} d^{3} k .
$$


By (3.8), (3.9), and (5.8), we have

$$
\frac{q^{2}}{4 \pi^{2}}\left(\log \frac{\Lambda}{\kappa}\right) \leq 2\left\langle\psi_{\kappa}, I \otimes N_{\mathrm{f}} \psi_{\kappa}\right\rangle_{\mathcal{H}}+2 \frac{q^{2}}{8 \pi^{2}} \Lambda^{2}\left\||x| \otimes I \psi_{\kappa}\right\|_{\mathcal{H}}^{2},
$$

which implies

$$
\left\{\frac{q^{2}}{8 \pi^{2}}\left(\log \frac{\Lambda}{\kappa}\right)-\frac{q^{2}}{8 \pi^{2}} \Lambda^{2}\left\||x| \otimes I \psi_{\kappa}\right\|_{\mathcal{H}}^{2}\right\} \leq\left\langle\psi_{\kappa}, I \otimes N_{\mathrm{f}} \psi_{\kappa}\right\rangle_{\mathcal{H}} .
$$

Therefore, (2.8) follows from (5.7) and (5.9).

\section{§6. Finite Uncertainty of Position in Ground State}

In this section, we show that if $H_{\kappa}^{\mathrm{N}}$ has a (normalized) ground state, then the uncertainty of the position in the ground state has to be finite. More precisely, if $H_{\kappa}^{\mathrm{N}}$ has a ground state $\psi_{\kappa}$, then $\psi_{\kappa} \in D\left(x^{2} \otimes I\right)$. Therefore, contrary to (2.7), we can indirectly prove that uncertainty of the position in the ground state is finite, $(\Delta x)_{\mathrm{gs}}<\infty$.

In the first half of this section, we consider the case where $V$ is in class (C1) and prove that if $H_{\kappa}^{\mathrm{N}}$ has a ground state $\psi_{\kappa}$, then $\psi_{\kappa}$ belongs to $D\left(x^{2} \otimes I\right)$. Moreover, to prove Theorem 2.4, we need a uniform estimate of $\left\||x| \otimes I \psi_{\kappa}\right\|_{\mathcal{H}}$ in the infrared cutoff $\kappa$. To do that we prepare some inequalities.

Lemma 6.1. Assume (A). Then, there exists a constant $C_{q}>0$ such that

$$
\sup _{0<\kappa<\Lambda}\left\|\left(H_{0}+I\right)\left(H_{\kappa}^{\mathrm{N}}-E_{\kappa}^{\mathrm{N}}+I\right)^{-1}\right\| \leq C_{q}
$$

Proof. For every $L^{2}\left(\mathbb{R}^{3}\right)$-valued function $f_{x}: \mathbb{R}_{x}^{3} \rightarrow L^{2}\left(\mathbb{R}^{3}\right)$ (i.e., $f_{x} \in$ $L^{2}\left(\mathbb{R}^{3}\right)$ for a.e. $x \in \mathbb{R}^{3}$ and $\left.\left\|f_{\star}\right\|_{L^{2}} \in L^{2}\left(\mathbb{R}^{3}\right)\right)$, we set $\left\|f_{\star}\right\|_{L^{2}, \infty}:=\operatorname{ess} \sup _{x \in \mathbb{R}^{3}}$ $\left\|f_{x}\right\|_{L^{2}}$. Combining fundamental inequalities for $H_{\mathrm{f}}$ and $\phi_{\kappa}(x)$ with an argument on the constant fiber direct integral (see, e.g., [3, Lemma 13-12]), for every $\varepsilon, \varepsilon^{\prime}>0$ and every $\psi \in D\left(H_{0}\right)$, we have

$$
\begin{aligned}
\left\|H_{\mathrm{I}, \kappa} \psi\right\|_{\mathcal{H}}^{2} \leq & (2+\varepsilon)\left\|\sqrt{2} \lambda_{\kappa, \star} / \sqrt{\omega}\right\|_{L^{2}, \infty}^{2}\left\|I \otimes H_{\mathrm{f}}^{1 / 2} \psi\right\|_{\mathcal{H}}^{2} \\
& +\frac{1}{2}\left(1+\frac{1}{2 \varepsilon}\right)\left\|\sqrt{2} \lambda_{\kappa, \star}\right\|_{L^{2}, \infty}^{2}\|\psi\|_{\mathcal{H}}^{2} \\
= & (2+\varepsilon)\left\|\sqrt{2} \lambda_{\kappa, 0} / \sqrt{\omega}\right\|_{L^{2}}^{2}\left\|I \otimes H_{\mathrm{f}}^{1 / 2} \psi\right\|_{\mathcal{H}}^{2} \\
& +\frac{1}{2}\left(1+\frac{1}{2 \varepsilon}\right)\left\|\sqrt{2} \lambda_{\kappa, 0}\right\|_{L^{2}}^{2}\|\psi\|_{\mathcal{H}}^{2}
\end{aligned}
$$


since $\left|e^{-i k x}\right|=1$. By fundamental inequalities, we have

$$
\begin{aligned}
\left\|I \otimes H_{\mathrm{f}}^{1 / 2} \psi\right\|_{\mathcal{H}}^{2} & =\left\langle\psi, I \otimes H_{\mathrm{f}} \psi\right\rangle_{\mathcal{H}} \leq\|\psi\|_{\mathcal{H}}\left\|I \otimes H_{\mathrm{f}} \psi\right\|_{\mathcal{H}} \\
& \leq \varepsilon^{\prime}\left\|\left(H_{0}+I\right) \psi\right\|_{\mathcal{H}}^{2}+\frac{1}{4 \varepsilon^{\prime}}\|\psi\|_{\mathcal{H}}^{2} .
\end{aligned}
$$

It follows from direct estimates that

$$
\left\|\lambda_{\kappa, 0}\right\|_{L^{2}}^{2} \leq \frac{\Lambda^{2}}{8 \pi^{2}}, \quad\left\|\lambda_{\kappa, 0} / \sqrt{\omega}\right\|_{L^{2}}^{2} \leq \frac{\Lambda}{4 \pi^{2}} .
$$

By $(6.2)-(6.4)$, we have

$$
\left\|H_{\mathrm{I}, \kappa} \psi\right\|_{\mathcal{H}} \leq C_{\Lambda}^{(1)}\left(\varepsilon, \varepsilon^{\prime}\right)\left\|\left(H_{0}+I\right) \psi\right\|_{\mathcal{H}}+C_{\Lambda}^{(2)}\left(\varepsilon, \varepsilon^{\prime}\right)\|\psi\|_{\mathcal{H}},
$$

where

$$
\begin{aligned}
C_{\Lambda}^{(1)}\left(\varepsilon, \varepsilon^{\prime}\right) & =\frac{\sqrt{\Lambda}}{2 \pi} \sqrt{2 \varepsilon^{\prime}(2+\varepsilon)}, \\
C_{\Lambda}^{(2)}\left(\varepsilon, \varepsilon^{\prime}\right) & =\frac{\sqrt{\Lambda}}{2 \pi} \sqrt{\frac{2+\varepsilon}{2 \varepsilon^{\prime}}+\frac{1}{2}\left(1+\frac{1}{2 \varepsilon}\right) \Lambda} .
\end{aligned}
$$

Since $\left(H_{0}+I\right) \psi=\left(H_{\kappa}^{\mathrm{N}}-E_{\kappa}^{\mathrm{N}}+I\right) \psi-q H_{\mathrm{I}, \kappa} \psi+\left(E_{\kappa}^{\mathrm{N}}-E_{\text {at }}\right) \psi$ for every $\psi \in D\left(H_{0}\right)$ and $\left|E_{\kappa}^{\mathrm{N}}-E_{\text {at }}\right| \leq q^{2}\left\|\lambda_{\kappa, 0}\right\|_{L^{2}}^{2}$ by Proposition 2.2 ,

$$
\begin{aligned}
\left\|\left(H_{0}+I\right) \psi\right\|_{\mathcal{H}} \leq & \frac{1}{1-|q| C_{\Lambda}^{(1)}\left(\varepsilon, \varepsilon^{\prime}\right)}\left\|\left(H_{\kappa}^{\mathrm{N}}-E_{\kappa}^{\mathrm{N}}+I\right) \psi\right\|_{\mathcal{H}} \\
& +\frac{|q| C_{\Lambda}^{(2)}\left(\varepsilon, \varepsilon^{\prime}\right)+q^{2} \Lambda^{2} / 8 \pi^{2}}{1-|q| C_{\Lambda}^{(1)}\left(\varepsilon, \varepsilon^{\prime}\right)}\|\psi\|_{\mathcal{H}}
\end{aligned}
$$

for every $\varepsilon, \varepsilon^{\prime}>0$ satisfying $1-|q| C_{\Lambda}^{(1)}\left(\varepsilon, \varepsilon^{\prime}\right)>0$, which implies

$$
\left\|\left(H_{0}+I\right)\left(H_{\kappa}^{\mathrm{N}}-E_{\kappa}^{\mathrm{N}}+I\right)^{-1}\right\| \leq \frac{1+|q| C_{\Lambda}^{(2)}\left(\varepsilon, \varepsilon^{\prime}\right)+q^{2} \Lambda^{2} / 8 \pi^{2}}{1-|q| C_{\Lambda}^{(1)}\left(\varepsilon, \varepsilon^{\prime}\right)} .
$$

We obtain the following lemma from Lemma 6.1.

Lemma 6.2. For every $q \neq 0$ and arbitrary $\kappa, \epsilon$ with $0<\epsilon$ and $0 \leq$ $\kappa<\Lambda$,

$$
\left\|\left(H_{0}+I\right)\left(H_{\kappa}^{\mathrm{N}}-E_{\kappa}^{\mathrm{N}}+\epsilon\right)^{-1}\right\| \leq \frac{C_{q}}{\min \{\epsilon, 1\}} .
$$


Proof. (6.6) follows from (6.1) and

$$
\left\|\left(H_{\kappa}^{\mathrm{N}}-E_{\kappa}^{\mathrm{N}}+I\right)\left(H_{\kappa}^{\mathrm{N}}-E_{\kappa}^{\mathrm{N}}+\epsilon\right)^{-1}\right\| \leq \begin{cases}\epsilon^{-1} & \text { if } \quad \epsilon<1 \\ 1 & \text { if } \quad \epsilon \geq 1\end{cases}
$$

Lemma 6.3. Let $V$ be in class $(\mathrm{C} 1)$.

(i) $D(V) \subset D\left(x^{2}\right)$.

(ii) If $\psi$ is in $D\left(H_{0}\right)$, then $\psi \in D(|x| \otimes I)$ and

$$
\||x| \otimes I \psi\|_{\mathcal{H}}^{2} \leq c_{1}\left\|H_{0}^{1 / 2} \psi\right\|_{\mathcal{H}}^{2}+c_{2}\|\psi\|_{\mathcal{H}}^{2} \leq c_{1}\left\|H_{0} \psi\right\|_{\mathcal{H}}^{2}+\left(c_{1}+c_{2}\right)\|\psi\|_{\mathcal{H}}^{2} .
$$

In particular, for $0 \leq \kappa \leq \Lambda$

$$
\left\||x| \otimes I \psi_{\kappa}\right\|_{\mathcal{H}}^{2} \leq\left(c_{1} C_{q}^{2}+c_{1}+c_{2}\right) .
$$

Proof. (i) directly follows from the first inequality of (C1-2). We obtain the first statement of (ii) by (C1-1), Proposition 2.2, and (i). As for the second statement, the first inequality of (6.7) is obtained in the same way as in $[2$, Lemma 4.6]. By Schwarz' inequality, we have

$$
\begin{aligned}
\left\|H_{0}^{1 / 2} \psi\right\|_{\mathcal{H}}^{2} & =\left\langle\psi, H_{0} \psi\right\rangle_{\mathcal{H}} \leq\|\psi\|_{\mathcal{H}}\left\|H_{0} \psi\right\|_{\mathcal{H}} \leq\left\|H_{0} \psi\right\|_{\mathcal{H}}^{2}+\frac{1}{4}\|\psi\|_{\mathcal{H}}^{2} \\
& \leq\left\|H_{0} \psi\right\|_{\mathcal{H}}^{2}+\|\psi\|_{\mathcal{H}}^{2}
\end{aligned}
$$

for $\psi \in D\left(H_{0}\right)$. So, we obtain the second inequality of (6.7). By (6.7), we have

$$
\begin{aligned}
\left\||x| \otimes I \psi_{\kappa}\right\|_{\mathcal{H}}^{2} \leq & c_{1}\left\|\left(H_{0}+I\right)\left(H_{\kappa}^{\mathrm{N}}-E_{\kappa}^{\mathrm{N}}+I\right)^{-1}\left(H_{\kappa}^{\mathrm{N}}-E_{\kappa}^{\mathrm{N}}+I\right) \psi_{\kappa}\right\|_{\mathcal{H}}^{2} \\
& +\left(c_{1}+c_{2}\right) \\
= & c_{1}\left\|\left(H_{0}+I\right)\left(H_{\kappa}^{\mathrm{N}}-E_{\kappa}^{\mathrm{N}}+I\right)^{-1} \psi_{\kappa}\right\|_{\mathcal{H}}^{2}+\left(c_{1}+c_{2}\right) .
\end{aligned}
$$

This inequality and Lemma 6.2 imply (6.8).

The following proposition follows from Lemma 6.3 directly:

Proposition 6.1 (finite uncertainty of position in ground state). Let $V$ be in class $(\mathrm{C} 1)$ and $\kappa$ satisfy $0 \leq \kappa<\Lambda$. If $H_{\kappa}^{\mathrm{N}}$ has a ground state $\psi_{\kappa}$, then $\psi_{\kappa} \in D\left(x^{2} \otimes I\right)$. Moreover, $\sup _{0<\kappa<\Lambda}|||x| \otimes I \psi_{\kappa} \|_{\mathcal{H}}<\infty$, provided that $\psi_{\kappa}$ exists for $0<\kappa<\Lambda$. 
Proof. Suppose that there exists a ground state $\psi_{\kappa}$ of $H_{\kappa}^{\mathrm{N}}$. Then, by (C11), Lemma 6.3(i), and Proposition 2.2, we have $\psi_{\kappa} \in D\left(H_{\kappa}^{\mathrm{N}}\right) \subset D\left(H_{\mathrm{at}} \otimes I\right) \subset$ $D\left(x^{2} \otimes I\right)$. The uniform estimate of $\left\||x| \otimes I \psi_{\kappa}\right\|_{\mathcal{H}}$ in $\kappa$ follows from (6.8) directly.

In the last half of this section, we consider the case where $V$ is in class $(\mathrm{C} 2)$ and we prove that if $H_{\kappa}^{\mathrm{N}}$ has a ground state $\psi_{\kappa}$, then $\psi_{\kappa}$ belongs to $D\left(x^{2} \otimes I\right)$. Moreover, we show a uniform estimate of $\left\||x| \otimes I \psi_{\kappa}\right\|_{\mathcal{H}}$ in the infrared cutoff $\kappa$, by proving the so-called exponential decay.

Let $E_{\kappa}^{\mathrm{N} V=0}=\inf \sigma\left(H_{\kappa}^{\mathrm{N} V=0}\right)$, where the superscript of $H_{\kappa}^{\mathrm{N} V=0}$ means that in (2.5) the external potential $V$ is omitted. The (positive) binding energy is defined by

$$
E_{\kappa}^{\mathrm{bin}}:=E_{\kappa}^{\mathrm{N} V=0}-E_{\kappa}^{\mathrm{N}} .
$$

The binding energy is bounded from below:

Proposition 6.2 (strict positivity of binding energy). Let $V$ be in class (C2). Fix $\kappa$ with $0 \leq \kappa<\Lambda$. Then,

$$
E_{\kappa}^{\text {bin }} \geq-E_{\text {at }}>0
$$

Proof. Using the idea proving [14, Theorem 3.1] for the Pauli-Fierz model, relation (6.9) was proved in [20, Proposition 4.4], but for the special external potential. It is easy to see that our proposition is also proven in the same way as in [20, Proposition 4.4] following the idea in the proof of $[14$, Theorem 3.1].

Proposition 6.3 (exponential decay). Fix $\kappa$ with $0 \leq \kappa<\Lambda$. Let $V$ be in class (C2). Assume $H_{\kappa}^{\mathrm{N}}$ has a (normalized) ground state $\psi_{\kappa}$. Then, there exist a sufficiently small $C_{0}>0$, a sufficiently large $N_{0} \in \mathbb{N}$, and $C>0$ such that $\psi_{\kappa} \in D\left(e^{C_{0}|x|}\right)$ and

$$
\begin{aligned}
& \left\|e^{C_{0}|x|} \psi_{\kappa}\right\|_{\mathcal{H}} \\
& \quad \leq e^{3 C_{0} N_{0}}\left\{1+C\left(\left|E_{\text {at }}\right|-\sup _{N_{0}<|x|}|V(x)|-C_{0}^{2}\right)^{-1 / 2}\right\},
\end{aligned}
$$

where

$$
\left|E_{\text {at }}\right|-\sup _{N_{0}<|x|}|V(x)|-C_{0}^{2}>0 .
$$


Proof. Since $\lim _{|x| \rightarrow \infty}|V(x)|=0$ in $(\mathrm{C} 2-1)$, we can take $N_{0} \in \mathbb{N}$ and $C_{0}>$ 0 such that (6.11) holds because we assumed $E_{\text {at }}<0$ in (C2-2). We take a nonnegative function $\mathbb{1}_{n} \in C_{0}^{\infty}(\mathbb{R})$ for each $n \in \mathbb{N}$ satisfying $\mathbb{1}_{n}(r)=1$ for $|r| \leq n$; $=0$ for $|r| \geq 3 n, 0 \leq \mathbb{1}_{n}(r) \leq 1$ for $n<|r|<3 n$. Since $\mathbb{1}_{n}^{\prime} \in C_{0}^{\infty}(\mathbb{R})$ again, we have $C_{n}:=\sup _{r}\left|d \mathbb{1}_{n}(r) / d r\right|<\infty$. We set $f_{\varepsilon}(r):=r(1+\varepsilon r)^{-1}$ for every $\varepsilon>0$ and $r \geq 0$. We define a function $G_{n, \varepsilon}(x)$ by $G_{n, \varepsilon}(x):=\left(1-\mathbb{1}_{n}(|x|)\right) f_{\varepsilon}\left(e^{C_{0}|x|}\right)$. Since $0 \leq f_{\varepsilon}(r) \leq \varepsilon^{-1}$ for all $r \geq 0$, the multiplication operators $f_{\varepsilon}\left(e^{C_{0}|x|}\right)$ and $G_{n, \varepsilon}$ are bounded on $L^{2}\left(\mathbb{R}^{3}\right)$. In the same way as in [20, Lemma 5.1], we have

$$
\begin{aligned}
E_{\kappa}^{\mathrm{bin}}\left\|G_{n, \varepsilon} \otimes I \psi_{\kappa}\right\|_{\mathcal{H}}^{2} \leq & \frac{1}{2}\left\langle\psi_{\kappa},\left|\nabla G_{n, \varepsilon}\right|^{2} \otimes I \psi_{\kappa}\right\rangle_{\mathcal{H}} \\
& +\sup _{n<|x|}|V(x)|\left\langle\psi_{\kappa}, G_{n, \varepsilon}^{2} \otimes I \psi_{\kappa}\right\rangle_{\mathcal{H}} .
\end{aligned}
$$

It is easy to check that

$$
\frac{\partial G_{n, \varepsilon}(x)}{\partial x_{j}}=-\frac{\partial \mathbb{1}_{n}(|x|)}{\partial x_{j}} f_{\varepsilon}\left(e^{C_{0}|x|}\right)+C_{0}\left(1-\mathbb{1}_{n}(|x|)\right) \frac{e^{C_{0}|x|}}{\left(1+\varepsilon e^{C_{0}|x|}\right)^{2}} \frac{x_{j}}{|x|} .
$$

So, using $\operatorname{supp} \mathbb{1}_{n}^{\prime} \subset[-3 n,-n] \cup[n, 3 n]$ and $\left(1+\varepsilon e^{C_{0}|x|}\right)^{-4}<\left(1+\varepsilon e^{C_{0}|x|}\right)^{-2}$, we have

$$
\begin{aligned}
\left|\nabla G_{n, \varepsilon}(x)\right|^{2} \leq & 2\left(\sup _{n \leq|x| \leq 3 n} f_{\varepsilon}\left(e^{C_{0}|x|}\right)\right)^{2} \sum_{j=1}^{3}\left(\frac{\partial \mathbb{1}_{n}(|x|)}{\partial x_{j}}\right)^{2} \\
& +2 C_{0}^{2}\left(1-\mathbb{1}_{n}(|x|)\right)^{2} \frac{e^{2 C_{0}|x|}}{\left(1+\varepsilon e^{C_{0}|x|}\right)^{4}} \\
\leq & 2\left(\frac{e^{3 C_{0} n}}{1+\varepsilon e^{3 C_{0} n}}\right)^{2} \sum_{j=1}^{3}\left(\frac{\partial \mathbb{1}_{n}(|x|)}{\partial x_{j}}\right)^{2}+2 C_{0}^{2} G_{n, \varepsilon}(x)^{2} \\
\leq & 2 e^{6 C_{0} n} \sum_{j=1}^{3}\left(\frac{\partial \mathbb{1}_{n}(|x|)}{\partial x_{j}}\right)^{2}+2 C_{0}^{2} G_{n, \varepsilon}(x)^{2}
\end{aligned}
$$

It is easy to check that

$$
\sum_{j=1}^{3}\left(\frac{\partial \mathbb{1}_{n}(|x|)}{\partial x_{j}}\right)^{2} \leq C_{n}^{2}
$$

By Proposition 6.2 and $(6.12)-(6.14)$, we have

$$
\begin{aligned}
& \left\|G_{N_{0}, \varepsilon} \otimes I \psi_{\kappa}\right\|_{\mathcal{H}}^{2} \\
& \quad \leq C_{N_{0}}^{2} e^{6 C_{0} N_{0}}\left\{\left|E_{\text {at }}\right|-\sup _{N_{0}<|x|}|V(x)|-C_{0}^{2}\right\}^{-1} .
\end{aligned}
$$


Let $d E_{|x|}(\xi)$ be the spectral measure of the multiplication operator $|x|$, i.e., the spectral representation of $|x|$ by $d E_{|x|}(\xi)$ is

$$
|x|=\int_{0}^{\infty} \xi d E_{|x|}(\xi) .
$$

Then, by Lebesgue's monotone convergence theorem and (6.15), we have

$$
\begin{aligned}
& C_{N_{0}}^{2} e^{6 C_{0} N_{0}}\left\{\left|E_{\text {at }}\right|-\sup _{N_{0}<|x|}|V(x)|-C_{0}^{2}\right\}^{-1} \\
& \geq \lim _{\varepsilon \downarrow 0}\left\|G_{N_{0}, \varepsilon} \otimes I \psi_{\kappa}\right\|_{\mathcal{H}}^{2} \\
& =\int_{0}^{\infty}\left(1-\mathbb{1}_{N_{0}}(\xi)\right)^{2} e^{2 C_{0} \xi} d\left\|E_{|x|}(\xi) \otimes I \psi_{\kappa}\right\|_{\mathcal{H}}^{2} \\
& =\left\|\left(1-\mathbb{1}_{N_{0}}(|x|)\right) e^{C_{0}|x|} \otimes I \psi_{\kappa}\right\|_{\mathcal{H}}^{2}
\end{aligned}
$$

with $\psi_{\kappa} \in D\left(\left(1-\mathbb{1}_{N_{0}}(|x|)\right) e^{C_{0}|x|} \otimes I\right)$. Moreover, since $\left|\mathbb{1}_{N_{0}}(|x|) e^{C_{0}|x|}\right| \leq$ $e^{3 C_{0} N_{0}}$, we have

$$
\left\|\mathbb{1}_{n}(|x|) e^{C_{0}|x|} \otimes I \psi_{\kappa}\right\|_{\mathcal{H}} \leq e^{3 C_{0} N_{0}}
$$

with $\psi_{\kappa} \in D\left(\mathbb{1}_{N_{0}}(|x|) e^{C_{0}|x|} \otimes I\right)$. Therefore, our statement that $\psi_{\kappa} \in$ $D\left(e^{C_{0}|x|}\right)$ and (6.10) follows from (6.16) and (6.17).

This exponential decay immediately implies the following.

Proposition 6.4 (finite uncertainty of position in ground state). Let $V$ be in class $(\mathrm{C} 2)$ and $\kappa$ satisfy $0 \leq \kappa<\Lambda$. If $H_{\kappa}^{\mathrm{N}}$ has a ground state $\psi_{\kappa}$, then $\psi_{\kappa} \in D\left(x^{2} \otimes I\right)$. Moreover, $\sup _{0<\kappa<\Lambda}\left\||x| \otimes I \psi_{\kappa}\right\|_{\mathcal{H}}<\infty$, provided that $\psi_{\kappa}$ exists for $0<\kappa<\Lambda$.

Proof. We have only to note the following. There exists $R_{0}>0$ such that $r \leq e^{C_{0} r}+R_{0}$ for every $r \geq 0$.

Proof of Theorem 2.2: $\quad$ Theorem 2.2 follows from Propositions 6.1 and 6.4 and Theorem 2.1.

Proof of Theorem 2.4: We note first that there exists a ground state $\psi_{\kappa}$ for $|q|<q_{\Lambda}$ and $0<\kappa<\Lambda$ by Proposition 2.3. Then, Theorem 2.4 follows from Propositions 6.1 and 6.4 and Theorem 2.3. 


\section{Acknowledgement}

The author thanks H. Spohn for hospitality at Technische Universität München. He also thanks V. Betz, F. Hiroshima, and J. Lőrinczi for giving him useful comments about their results in Munich. He is grateful to V. Bach for hospitality at Johannes Gutenberg Universität Mainz. It is his pleasure to thank to M. Griesemer for giving advice on spatial localization, and to Z. Ammari and A. Pizzo for discussing Nelson's model in Mainz. He is grateful to A. Arai for his comments on this paper, and to J. Dereziński and C. Gérard for information about their recent results. He is deeply grateful to the referee for many thoughtful comments, which helped him to correct many errors in the original manuscript and to reformulate Theorems 2.1 and 2.2 in a general framework. In particular, the author could notice the physical image lying idle in the original manuscript, which is stated in $\S 1$, through the communications with the referee. Based on this image he could complete the reformations of them.

\section{References}

[1] Ammari, Z., Asymptotic completeness for a renormalized nonrelativistic Hamiltonian in quantum field theory: The Nelson model, Math. Phys. Anal. Geom., 3 (2000), 217-285.

[2] Arai, A., Ground state of the massless Nelson model without infrared cutoff in a nonFock representation, Rev. Math. Phys., 13 (2001), 1075-1094.

[3] Fock Spaces and Quantized Field. II (in Japanese), Nihon-hyōron-sha, Tokyo, 2000 .

[4] Arai, A. and Hirokawa, M., On the existence and uniqueness of ground state of a generalized spin-boson model, J. Funct. Anal., 151 (1997), 455-503.

[5] - Ground states of a general class of quantum field Hamiltonians, Rev. Math. Phys., 12 (2000), 1085-1135.

[6] Arai, A., Hirokawa, M. and Hiroshima, F., On the absence of eigenvectors of Hamiltonians in a class of massless quantum field models without infrared cutoffs, J. Funct. Anal., 168 (1999), 470-497.

[7] $\ldots$, Regularity of ground states in quantum field models, preprint 2004, arXiv:math$\mathrm{ph} / 0409055$.

[8] Bach, V., Fröhlich, J. and Sigal, I. M., Spectral analysis for systems of atoms and molecules coupled to the quantized radiation field, Comm. Math. Phys., 207 (1999), 249-290.

[9] Betz, V., Hiroshima, F., Lőrinczi, J., Minlos, R. A. and Spohn, H., Ground state properties of the Nelson Hamiltonian: a Gibbs measure-based approach, Rev. Math. Phys., 14 (2002), 173-198.

[10] Bloch, F. and Nordsieck, A., Notes on the radiation field of the electron, Phys. Rev., 52 (1937), 54-59.

[11] Dereziński, J. and Gérard, C., Scattering theory of infrared divergent Pauli-Fierz Hamiltonians, Ann. Henri Poincaré, 5 (2004), 523-577.

[12] Fröhlich, J., On the infrared problem in a model of scalar electrons and massless, scalar bosons, Ann. Inst. H. Poincaré Sect. A (N.S.), 19 (1973), 1-103. 
[13] Gérard, C., On the existence of ground states for massless Pauli-Fierz Hamiltonians, Ann. Henri Poincaré, 1 (2000), 443-459.

[14] Griesemer, M., Lieb, E. H. and Loss, M., Ground states in non-relativistic quantum electrodynamics, Invent. Math., 145 (2001), 557-595.

[15] Griesemer, M., Exponential decay and ionization thresholds in non-relativistic quantum electrodynamics, J. Funct. Anal., 210 (2004), 321-340.

[16] Hainzl, C., Hirokawa, M. and Spohn, H., Binding energy for hydrogen-like atom in the Nelson model without cutoffs, J. Funct. Anal., 220 (2005), 424-459.

[17] Hirokawa, M., Mathematical Addendum for "Infrared Catastrophe for Nelson's Model" (mp_arc 03-512), preprint 2003, mp_arc 03-551.

[18] Hirokawa, M., Recent developments in mathematical methods for models in nonrelativistic quantum electrodinamics, A Garden of Quanta. Essays in Honor of Hiroshi Ezawa (Arafune, J., Arai, A., Kobayashi, M., Nakamura, K., Nakamura, T., Ojima, I., Sakai, N., Tonomura, A. and Watanabe, K., eds.), 209-242, World Scientific, 2003.

[19] Hirokawa, M., A Mathematical Mechanism of Infrared Catastrophe, preprint 2004, mp_arc 04-83, arXiv:math-ph/0403008.

[20] Hirokawa, M., Hiroshima, F. and Spohn, H., Ground state for point particles interacting through a massless scalar bose field, Adv. Math., 191 (2005), 339-392.

[21] Hiroshima, F., Multiplicity of ground states in quantum field models: applications of asymptotic fields, J. Funct. Anal., 224 (2005), 431-470.

[22] Høegh-Krohn, R., Asymptotic fields in some models of quantum field theory, I, J. Math. Phys., 9 (1968), 2075-2080.

[23] Kato, Y. and Mugibayashi, N., Regular perturbation and asymptotic limits of operators in quantum field theory, Prog. Theoret. Phys., 30 (1963), 103-133.

[24] Lörinczi, J., Minlos, R. A. and Spohn, H., The infrared behaviour in Nelson's model of a quantum particle coupled to a massless scalar field, Ann. Henri Poincaré, 3 (2002), 269-295.

[25] Nelson, E., Interaction of nonrelativistic particles with a quantized scalar field, J. Math. Phys., 5 (1964), 1190-1197.

[26] Pauli, W. and Fierz, M., Zur Theorie der Emission langwelliger Lichtquanten, Nuovo Cimento, 15 (1938), 167-187.

[27] Pizzo, A., One particle (improper) states and scattering states in Nelson's massless model, Ann. Henri Poincaré, 4 (2003), 439-486.

[28] Reed, M. and Simon, B., Methods of Modern Mathematical Physics I: Functional Analysis, Academic Press, San Diego, 1980.

[29] - Methods of Modern Mathematical Physics II: Fourier Analysis, Selfadjointness, Academic Press, San Diego, 1980.

[30] $\longrightarrow$ Methods of Modern Mathematical Physics IV: Analysis of Operators, Academic Press, San Diego, 1978.

[31] Spohn, H., Ground state of quantum particle coupled to a scalar boson field, Lett. Math. Phys., 44 (1998), 9-16. 